\title{
Fruit and Vegetable Intake and Mental Health in Adults: A Systematic Review
}

\author{
Dominika Głąbska $^{1} *\left(\mathbb{D}\right.$, Dominika Guzek ${ }^{2}$, Barbara Groele $^{1}$ and Krystyna Gutkowska ${ }^{2}$ (D) \\ 1 Department of Dietetics, Institute of Human Nutrition Sciences, Warsaw University of Life \\ Sciences (SGGW-WULS), 159C Nowoursynowska Street, 02-787 Warsaw, Poland; barbara_groele@sggw.pl \\ 2 Department of Food Market and Consumer Research, Institute of Human Nutrition Sciences, Warsaw \\ University of Life Sciences (SGGW-WULS), 159C Nowoursynowska Street, 02-787 Warsaw, Poland; \\ dominika_guzek@sggw.pl (D.G.); krystyna_gutkowska@sggw.pl (K.G.) \\ * Correspondence: dominika_glabska@sggw.pl; Tel.: +48-22-593-71-26
}

Received: 15 November 2019; Accepted: 28 December 2019; Published: 1 January 2020

\begin{abstract}
The role of a properly balanced diet in the prevention and treatment of mental disorders has been suggested, while vegetables and fruits have a high content of nutrients that may be of importance in the case of depressive disorders. The aim of the study was to conduct a systematic review of the observational studies analyzing association between fruit and vegetable intake and mental health in adults. The search adhered to the guidelines of Preferred Reporting Items for Systematic Reviews and Meta-Analyses (PRISMA), and the review was registered in the International Prospective Register of Systematic Reviews (PROSPERO) database (CRD42019138148). A search for peer-reviewed observational studies published until June 2019 was performed in PubMed and Web of Science databases, followed by an additional manual search for publications conducted via analyzing the references of the found studies. With respect to the intake of fruit and/or vegetable, studies that assessed the intake of fruits and/or vegetables, or their processed products (e.g., juices), as a measure expressed in grams or as the number of portions were included. Those studies that assessed the general dietary patterns were not included in the present analysis. With respect to mental health, studies that assessed all the aspects of mental health in both healthy participants and subjects with physical health problems were included, but those conducted in groups of patients with intellectual disabilities, dementia, and eating disorders were excluded. To assess bias, the Newcastle-Ottawa Scale (NOS) was applied. A total of 5911 studies were independently extracted by 2 researchers and verified if they met the inclusion criteria using a 2-stage procedure (based on the title, based on the abstract). After reviewing the full text, a total of 61 studies were selected. A narrative synthesis of the findings from the included studies was performed, which was structured around the type of outcome. The studies included mainly focused on depression and depressive symptoms, but also other characteristics ranging from general and mental well-being, quality of life, sleep quality, life satisfaction, flourishing, mood, self-efficacy, curiosity, creativity, optimism, self-esteem, stress, nervousness, or happiness, to anxiety, minor psychiatric disorders, distress, or attempted suicide, were analyzed. The most prominent results indicated that high total intake of fruits and vegetables, and some of their specific subgroups including berries, citrus, and green leafy vegetables, may promote higher levels of optimism and self-efficacy, as well as reduce the level of psychological distress, ambiguity, and cancer fatalism, and protect against depressive symptoms. However, it must be indicated that the studies included were conducted using various methodologies and in different populations, so their results were not always sufficiently comparable, which is a limitation. Taken together, it can be concluded that fruits and/or vegetables, and some of their specific subgroups, as well as processed fruits and vegetables, seems to have a positive influence on mental health, as stated in the vast majority of the included studies. Therefore, the general recommendation to consume at least 5 portions of fruit and vegetables a day may be beneficial also for mental health.
\end{abstract}


Keywords: fruits; vegetables; juices; intake; mental health; mental disorders; depression; anxiety; well-being; quality of life

\section{Introduction}

Mental disorders (referred by the International Statistical Classification of Diseases and Related Health Problems (ICD-10) [1] as F00-F99) are indicated as a global problem by the World Health Organization (WHO). One in four individuals suffers from a mental disorder during some period of life [2], and hence, the WHO has recognized mental disorders as a health burden that should be no longer neglected [3]. Within the Comprehensive Mental Health Action Plan 2013-2020 implemented by the WHO [4], it was indicated that there is a need for, inter alia, evidence-based practice for a life course and a multidisciplinary approach to solve this problem. Taking into account the recommendation of multisectoral actions that should be taken by various specialists from the sectors of health and social services [4], the potential influence of nutrition should be included.

Some published studies have concluded that following a properly balanced diet allows maintaining a better well-being and lowers the risk of mental disorders in adolescents and students [5,6]. This finding was also confirmed by $\mathrm{O}^{\prime} \mathrm{Neil}$ et al. in their systematic review [7]. At the same time, a therapeutic role was indicated for the Mediterranean diet in 2 randomized controlled trials-HELFIMED [8] and SMILES [9] - for the adults suffering from depression.

However, it is not easy to assess single elements of a whole dietary pattern and recognize their effect, as each individual consumes a wide range of various products at the same time [10]. Nonetheless, some attempts have been made to identify the elements of a properly balanced diet, which are also typical for the Mediterranean diet, that may be defined as food products with beneficial effects on mental health and aiding in recovery from mental illness; these include fish and seafood, legumes, leafy greens and other vegetables, olive oil, dairy beverages, and nuts [11]. Similarly, the highest Antidepressant Food Score (AFS), calculated based on the content of 12 nutrients that are related to the prevention and treatment of depressive disorders, was given for vegetables, followed by organ meats and fruits [12].

So far, some reviews have assessed the influence of the intake of fruit and vegetable, including a meta-analysis by Liu et al. [13], which focused exclusively on the risk of depression and aroused a great deal of controversy [14,15], and a recent meta-analysis by Saghafian et al. [16], which also focused exclusively on the risk of depression. In addition, a systematic review of prospective research, analyzing the effect on psychological health, was performed by Tuck et al. [17], but it focused exclusively on vegetables. A review by Rooney et al. [18] analyzed the role of fruits and vegetables in the broad aspects of psychological well-being, but it was not a systematic review and the authors emphasized the need for a more exhaustive synthesis of studies in the form of a systematic review.

Fruit and vegetables are beneficial for general health and the recent studies indicate that they may be even more important than it was previously supposed and that to obtain prevention of cardiovascular disease, cancer, and premature mortality even the higher intake than the generally-recommended $400 \mathrm{~g}$ is needed [19]. However, the mechanism of their influence on mental health is still unknown, while a number of possible factors that may contribute to the positive impact are indicated [18]. Among them, there are specific nutrients, which are known as such that may be related to mental health and for which fruit and vegetables are indicated as a valuable sources in diet, such as complex carbohydrates [20] and fiber [21], being associated with glycemic index [22], C vitamin [23], B vitamins [24], carotenoids [25], potassium [26], and polyphenols [27]. The other explanations are associated with either possibility of reverse mechanism (higher level of mental health may promote better diet, including higher fruit and vegetables intake) [28], or psychological explanation (following better diet, including higher fruit and vegetables intake may promote more positive emotions and better mental health) [29]. 
Taking this into account, the present study aimed to conduct a systematic review of the observational studies analyzing the association between the intake of fruit and vegetables and the broad aspects of mental health in adults.

\section{Materials and Methods}

\subsection{Study Design}

The search used Preferred Reporting Items for Systematic Reviews and Meta-Analyses (PRISMA) guidelines [30] and the review was registered in International Prospective Register of Systematic Reviews (PROSPERO) database (CRD42019138148). A systematic review of peer-reviewed observational studies published until June 2019 was performed in PubMed and Web of Science databases with the additional manual search conducted via references of found studies.

\subsection{Inclusion/Exclusion Criteria}

The present systematic review analyzed the association between the intake of fruit and/or vegetable and mental health in adults. The studies included were those that assessed the intake of fruit or vegetables (conducted using various methods) to specify the amount consumed (supposed reason), together with an assessment of mental health (various factors, both self-assessed and diagnosed) (supposed consequence) and an analysis of the association between the indicated factors. The studies which analyzed the reverse association (assessment of the intake of fruit or vegetables as influenced by mental health) were not included, but those that did not define the supposed reason and consequence and considered only the intake of fruit or vegetables and mental health as coexisting factors were included.

With respect to the intake of fruit and/or vegetables, studies that assessed the intake of fruits and/or vegetables, or their processed products (e.g., juices), either as a measure expressed in grams or as the number of portions, were included. Studies involving the analysis of highly processed products (e.g., jam, beverages other than juices) were excluded. Only those studies presenting the observations of habitual intake, with no short-term interventions, were included. The studies that did not assess single products (intake of a specific fruit or vegetable) or groups (total intake of fruits and/or vegetables) but assessed the general dietary patterns were analyzed; however, they were not included in the present analysis if there was no separate assessment of the intake of fruit or vegetables.

With respect to mental health, studies that assessed all the aspects of mental health in both healthy participants and those with physical health problems were included. However, studies conducted in groups of patients with (1) intellectual disabilities, (2) dementia, and (3) eating disorders were excluded. Only studies conducted in humans and especially adults were included.

The included studies presented the research conducted in all the countries, independent of the location and income, but only those published in English, in peer-reviewed journals were taken into account.

\subsection{Search Strategy}

The search for studies was performed in PubMed and Web of Science databases; the strategy applied for electronic search in both databases is presented in Supplementary Table S1. In addition, a manual search for publications was conducted via analyzing the references of the found studies. The search focused on gathering observational studies published until June 2019.

Studies were independently extracted by 2 researchers and screened based on the inclusion and exclusion criteria using a 2-stage procedure: (1) Studies were verified based on the title; and (2) studies included based on the title were verified based on the abstract. Any disagreement between the two researchers over including a particular study was resolved through discussion with a third researcher. The full texts of these potentially eligible studies were retrieved (by asking the corresponding author for the full text, if needed) and independently assessed for eligibility by the two researchers. Again, 
any disagreement between them over the eligibility of a particular study was resolved through discussion with a third researcher.

The procedure of identification, screening, assessment of eligibility, and inclusion is presented in Figure 1.

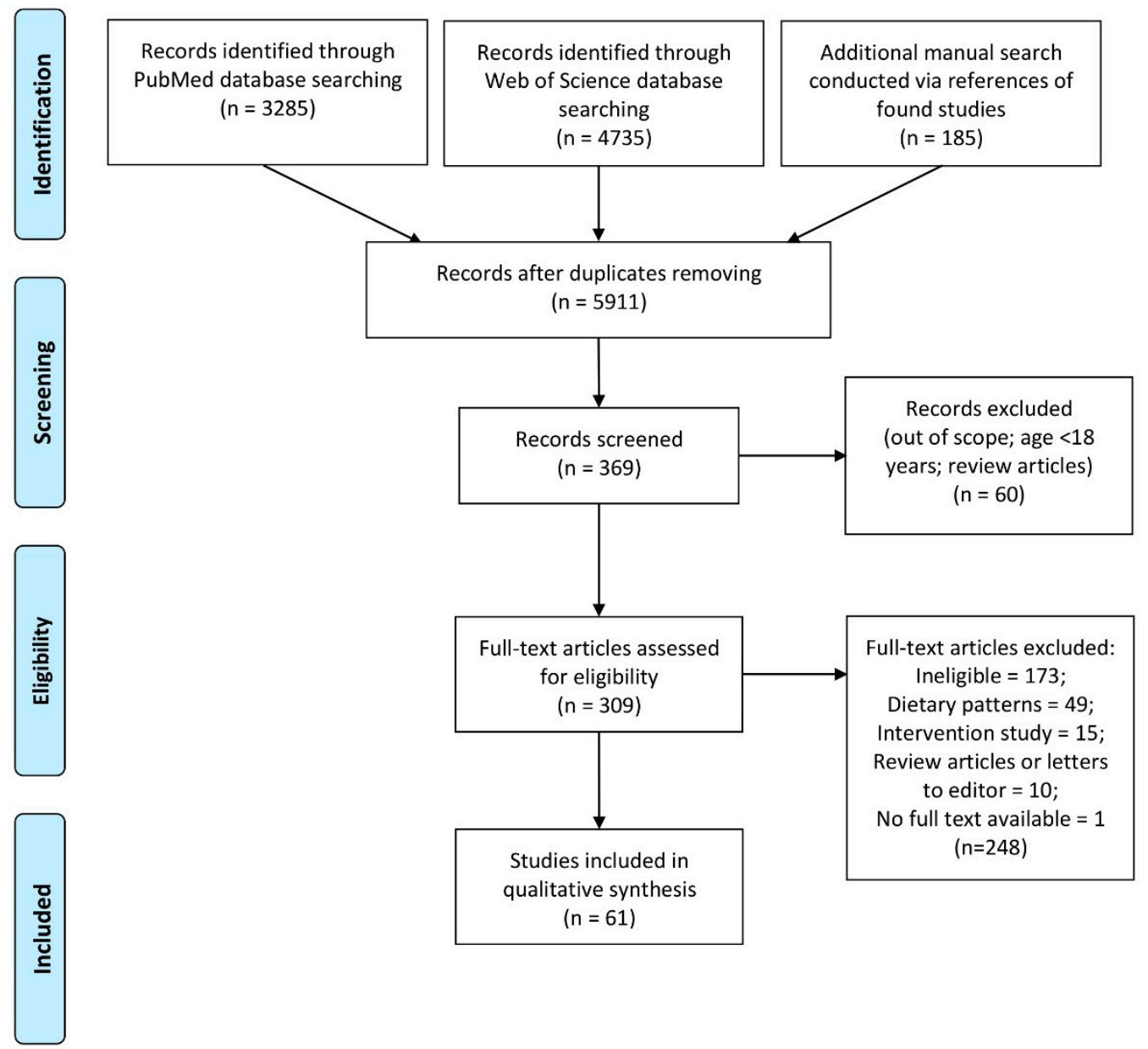

Figure 1. Procedure of identification, screening, eligibility assessment, and inclusion within the conducted systematic review.

\subsection{Data Extraction}

Data extraction was carried out independently by two researchers. Any disagreement between them over specific data was resolved through discussion with a third researcher. Missing data were requested from the authors of the studies, if possible, while the total number of 46 individual e-mails have been sent to corresponding authors of the included studies, as well as to first authors (if the first author was not corresponding one, but his e-mail address was provided in the full text of article). For 6 studies, authors not only answered, they also provided the requested data (in tables those data are referred as provided by authors on request).

Data extracted from the studies included the following: General details of the study (author, study design), observation (country/location, study group, time), participants (number of participants, gender proportions, age, inclusion criteria, exclusion criteria), exposure (method of assessment, measure of fruit and vegetable, other fruit and vegetable products included), outcomes (method of assessment, psychological measure), and findings (observations, conclusions).

In order to assess bias and the general quality of the studies, based on the Cochrane recommendations for the tools for assessing the methodological quality or the risk of bias in non-randomized studies [31], the Newcastle-Ottawa Scale (NOS) [32] was applied, which is used 
commonly [33]. Each included study was assessed for the following criteria: For case-control studies-selection (scale from 0 to 4), comparability (scale from 0 to 2), and exposure (scale from 0 to 3); and for cohort studies - selection (scale from 0 to 4), comparability (scale from 0 to 2), and outcome (scale from 0 to 3). The results were interpreted based on the commonly assumed criteria and attributed to the following categories: very high risk of bias (0-3 NOS points), high risk of bias (4-6 NOS points), and low risk of bias (7-9 NOS points) [34].

The following types of outcomes were included to the presented systematic review: General and mental well-being, quality of life, sleep quality, life satisfaction, and mood (general outcomes); flourishing, self-efficacy, curiosity, creativity, optimism, self-esteem, and happiness (positive outcomes); stress, nervousness, anxiety, minor psychiatric disorders, distress, depressive symptoms, depression, and attempted suicide (negative outcomes). Due to the fact that numerous various outcomes were included, while the number of studies for each outcome differed from only one study, to a lot of studies (as for depression or depressive symptoms), it was not possible to summarize the results in the form of meta-analysis, as it requires including comparable studies only (for one type of outcome). As not only outcomes, but also way to express risk factor (namely fruit and/or vegetable intake), studied populations, and settings were not comparable, the studies may not be treated as sufficiently similar to be able to reanalyze the data in the form of meta-analysis, but in the future, while the number of such studies will be higher, for each outcome, an adequate meta-analysis will be valuable to conclude. Taking it into account, based on the data extracted and the assessment of the general quality of the study, a narrative synthesis of the findings from the included studies was performed, which was structured around the type of outcome.

\section{Results}

The list of studies included to the systematic review is presented in Supplementary Table S2. The basic study details and design of observation for the studies included to the systematic review is presented in Table 1. The studies presented in all the tables are listed accordingly based on the year of publication. Among 61 included studies, the majority were conducted for European countries (20 studies), Asian countries (14 studies), or United States of America (USA) (11 studies), but also those conducted for African (1 study), or South American country (1 study), Australia or New Zealand (7 studies), or Canada (3 studies) were included and some of them were conducted for mixed countries (4 studies). The studied populations were mainly adults (including young, middle aged, and older ones, or only some of indicated groups) (37 studies), as well as only young ones (12 studies), middle aged ones ( 3 studies), or old ones (9 studies). It should be indicated that 3 included studies were conducted in a specific populations, while the health-related inclusion criteria for the study were defined as prediabetes and/or prehypertension [35], being after coronary artery bypass grafting surgery [36], or having excessive body mass [37].

The characteristics of the study participants for the studies included to the systematic review is presented in Supplementary Table S3. The characteristics of the study exposure and outcomes for the studies included to the systematic review is presented in the Table 2. Among 61 included studies, for the majority of them, a food frequency questionnaire, or a rapid screener was applied (32 studies), or a specific questionnaire to assess the diet quality (1 study), or a simple question about fruit and vegetable intake (22 studies), but for 4 studies, the method of dietary recall or record was applied, either alone or combined with previously indicated methods, and for 2 studies the applied method was not specified. To assess the mental health, various aspects of it were analyzed in the studies, while either a single aspect or some aspects combined were assessed, including mainly depressive symptoms (18 studies), depression (15 studies), general and mental well-being (9 studies), stress (8 studies), distress (8 studies), quality of life (7 studies), mood (5 studies), or anxiety (5 studies), but also in some of them happiness (4 studies), life satisfaction (3 studies), or optimism (2 studies) were analyzed, and in single studies only: sleep quality, flourishing, self-efficacy, curiosity, creativity, self-esteem, nervousness, minor psychiatric disorders, or attempted suicide were indicated. 
The characteristics of the study findings for the studies included to the systematic review accompanied by the quality assessment based on the total score for the Newcastle-Ottawa Scale is presented in the Table 3. The detailed results of the quality assessment based on the total score for the Newcastle-Ottawa Scale for categories of selection, comparability and exposure/outcome are presented in Supplementary Table S4. Among 61 included studies, for the majority of them, a statistically significant influence of fruit and/or vegetable consumption on mental health was proven, but there were also some studies, for which it was proven only in case of some factors associated with mental health and not for all assessed ones and only few studies, for which it was not proven that there is any association [37-39]. While concluding, authors of the included studies emphasized the existing association, but also among most important remarks, they indicated risks resulting from not following a recommendation to consume at least 5 portions of fruit and vegetable each day (2 studies), or they indicated especially beneficial groups of fruit and vegetables ( 2 studies). 
Table 1. General details of the study and design for the studies included to the systematic review.

\begin{tabular}{|c|c|c|c|c|c|}
\hline \multirow{2}{*}{ Ref. } & \multicolumn{3}{|c|}{ Study Details } & \multicolumn{2}{|c|}{ Observation } \\
\hline & Author (Year) & Study Design & Country/Location & Study Group & Time \\
\hline [40] & Chang et al., 2019 & $\begin{array}{l}\text { Cross-sectional study within Special Supplemental Nutrition } \\
\text { Program for Women, Infants, and Children in Michigan, USA }\end{array}$ & United States of America (USA) & Non-Hispanic women & May to August 2010 \\
\hline [41] & Cheng et al., 2019 & Observational study & China/Linyi, Shandong Province & $\begin{array}{l}\text { Middle-aged Chinese } \\
\text { population }\end{array}$ & May 2016 to June 2017 \\
\hline [42] & Gehlich et al., 2019 & $\begin{array}{l}\text { Longitudinal population-based study within Survey of Health, } \\
\text { Ageing and Retirement in Europe (SHARE) }\end{array}$ & $\begin{array}{l}\text { Austria, Belgium, Denmark, France, } \\
\text { Germany, Italy, Netherlands, Slovenia, Spain, } \\
\text { Sweden, Switzerland }\end{array}$ & Older adults & $\begin{array}{l}2011 \text { and } 2013 \text { waves of the } \\
\text { study }\end{array}$ \\
\hline [43] & Gehlich et al., 2019 & $\begin{array}{l}\text { Cross-sectional, population-based study based on the WHO } \\
\text { Study on Global Ageing and Adult Health (SAGE) }\end{array}$ & $\begin{array}{l}\text { China, India, Mexico, Russia, South Africa, } \\
\text { Ghana }\end{array}$ & Adults $\geq 50$ years & $2007-2010$ \\
\hline [44] & Goh et al., 2019 & $\begin{array}{l}\text { Cross-sectional, population-based study within Well-being of } \\
\text { the Singapore Elderly (WiSE) Study }\end{array}$ & Singapore & Adults $\geq 60$ years & December 2013 \\
\hline [45] & Ocean et al., 2019 & $\begin{array}{c}\text { Longitudinal study within the UK Household Longitudinal } \\
\text { Survey (UKHLS) }\end{array}$ & United Kingdom (UK) & General population & 2010-2017 waves of the study \\
\hline [35] & Pengpid et al., 2019 & Longitudinal study within a lifestyle intervention trial & Thailand/Nakhon Pathom province & $\begin{array}{l}\text { Temple members with } \\
\text { prediabetes and/or } \\
\text { prehypertension }\end{array}$ & 2016-2018 \\
\hline [46] & Salvatore et al., 2019 & Cross-sectional study $-10,001$ Dalmatians Study & Croatia/Split and Island of Korčula & Adults & 2007-2015 \\
\hline [47] & Azupogo et al., 2018 & Cross-sectional study & Ghana/Tolon and Savelugu Districts & Rural women in fertile age & April to May 2016 \\
\hline [48] & Baharzadeh et al., 2018 & Cross-sectional study & Iran/Khorramabad & $\begin{array}{l}\text { Women attending health } \\
\text { centers }\end{array}$ & May to October 2017 \\
\hline [49] & Boehm et al., 2018 & $\begin{array}{c}\text { Observational population-based study within English } \\
\text { Longitudinal Study of Ageing (ELSA) }\end{array}$ & UK & Adults $\geq 50$ years & 2006-2013 waves of the study \\
\hline [50] & Brookie et al., 2018 & Cross-sectional study & New Zealand, USA & $\begin{array}{l}\text { Young adults aged } 18-25 \\
\text { recruited as part of psychology } \\
\text { course at university, or through } \\
\text { an online crowdsourcing } \\
\text { marketplace }\end{array}$ & March to June 2017 \\
\hline [51] & Hoare et al., 2018 & Longitudinal, population-based study & USA & $\begin{array}{l}\text { Adolescents at baseline, adults } \\
\text { in follow-up }\end{array}$ & $\begin{array}{l}1994-1995 \text { and } 2007-2008 \\
\text { waves of the study }\end{array}$ \\
\hline [52] & Jyväkorpi et al., 2018 & $\begin{array}{l}\text { Cross-sectional study in longitudinal Helsinki Businessmen } \\
\text { Study (HBS) cohort }\end{array}$ & Finland/Helsinki & Oldest-old, home-dwelling men & 2016 \\
\hline [53] & Pagliai et al., 2018 & Cross-sectional study-Mugello Study & Italy/Florence & Nonagenarians (90-99 years) & Not specified \\
\hline [54] & Saghafian et al., 2018 & $\begin{array}{l}\text { Cross-sectional study within Study on the Epidemiology of } \\
\text { Psychological, Alimentary Health and Nutrition (SEPAHAN) }\end{array}$ & Iran & $\begin{array}{l}\text { Adults working in health } \\
\text { centers }\end{array}$ & $2010^{1}$ \\
\hline [55] & Tan et al., 2018 & Cross-sectional study & Germany, Netherlands & Adults $\geq 20$ years & 2013-2015 \\
\hline
\end{tabular}


Table 1. Cont.

\begin{tabular}{|c|c|c|c|c|c|}
\hline \multirow{2}{*}{ Ref. } & \multicolumn{3}{|c|}{ Study Details } & \multicolumn{2}{|c|}{ Observation } \\
\hline & Author (Year) & Study Design & Country/Location & Study Group & Time \\
\hline [56] & Welch and- Ellis 2018 & $\begin{array}{c}\text { Cross-sectional, population-based study - Health Information } \\
\text { National Trends Survey (HINTS) }\end{array}$ & USA & Adults & 2011-2017 waves of the study \\
\hline [57] & Bishwajit et al., 2017 & Cross-sectional study based on World Health Survey of WHO & Bangladesh, India, Nepal & Adults & 2002-2004 \\
\hline [58] & Nguyen et al., 2017 & $\begin{array}{l}\text { Longitudinal, cross-sectional, population-based study-Sax } \\
\text { Institute's } 45 \text { and Up Study }\end{array}$ & Australia/New South Wales & Adults & 2006-2008, 2010 \\
\hline [59] & $\begin{array}{l}\text { Peltzer and Pengpid } \\
\qquad 2017\end{array}$ & Cross-sectional study & $\begin{array}{l}\text { Bangladesh, Barbados, Cameroon, China, } \\
\text { Colombia, Egypt, Grenada, India, Indonesia, } \\
\text { Ivory Coast, Jamaica, Kyrgyzstan, Laos, } \\
\text { Madagascar, Malaysia, Mauritius, Namibia, } \\
\text { Nigeria, Pakistan, Philippines, Russia, } \\
\text { Singapore, South Africa, Thailand, Tunisia, } \\
\text { Turkey, Venezuela, Vietnam }\end{array}$ & University students & Not specified \\
\hline [60] & Ribeiro et al., 2017 & $\begin{array}{c}\text { Cross-sectional and longitudinal study-African American } \\
\text { Health (AAH) }\end{array}$ & USA/Missouri, St. Louis & $\begin{array}{l}\text { Urban-dwelling African } \\
\text { Americans }\end{array}$ & 2007-2010 \\
\hline [61] & Richard et al., 2017 & $\begin{array}{l}\text { Cross-sectional, population-based study—based on COhorte } \\
\text { LAUSannoise(CoLaus)/Psychiatric CoLaus (PsyCoLaus) and } \\
\text { Swiss Health Survey (SHS) }\end{array}$ & Switzerland & Adults $\geq 40$ years & 2009-2012 \\
\hline [62] & Warner et al., 2017 & Cross-sectional study & USA/New England & University students & 2013-2014 \\
\hline [63] & Wolniczak et al., 2017 & $\begin{array}{l}\text { Observational, population-based study within Health } \\
\text { Questionnaire of the Demographic Health Survey-Encuesta } \\
\text { Demográfica y de Salud Familiar (ENDES) }\end{array}$ & Peru & Adults & 2014 \\
\hline [64] & Chi et al., 2016 & $\begin{array}{l}\text { Longitudinal study-Taiwan Longitudinal Survey on Aging } \\
\text { (TLSA) }\end{array}$ & Taiwan & Adults $\geq 53$ year & 1999,2003 \\
\hline [65] & Lesani et al., 2016 & Cross-sectional study & Iran & $\begin{array}{l}\text { Students of Qazvin University } \\
\text { of Medical Sciences }\end{array}$ & Not specified \\
\hline [66] & $\begin{array}{l}\text { Mujcic and Oswald } \\
2016\end{array}$ & $\begin{array}{l}\text { Longitudinal, population-based study-Household, Income, } \\
\text { and Labour Dynamics in Australia (HILDA) Survey }\end{array}$ & Australia & $\begin{array}{l}\text { Adolescents or adults at } \\
\text { baseline, adults in follow-up }\end{array}$ & $2007,2009,2013$ \\
\hline [67] & Beezhold et al., 2015 & Observational study & USA, Canada and other countries ( $16 \%)$ & Adults & 2013 \\
\hline [68] & Conner et al., 2015 & Micro-longitudinal study within Daily Life Study & New Zealand & $\begin{array}{l}\text { Students at the University of } \\
\text { Otago }\end{array}$ & 2013 \\
\hline [69] & Kingsbury et al., 2015 & $\begin{array}{c}\text { Longitudinal population-based study-National Population } \\
\text { Health Survey (NPHS) }\end{array}$ & Canada & Adults & $2002-2011$ \\
\hline [70] & Kwon et al., 2015 & $\begin{array}{l}\text { Observational study within Racial and Ethnic Approaches to } \\
\text { Health Across the United States (REACH US) }\end{array}$ & USA/New York & $\begin{array}{l}\text { Minority groups in ethnic } \\
\text { enclaves }\end{array}$ & 2009-2012 \\
\hline [71] & Papier et al., 2015 & Cross-sectional study & Australia & $\begin{array}{l}\text { First year undergraduate } \\
\text { students of Griffith University }\end{array}$ & 2012-2013 \\
\hline
\end{tabular}


Table 1. Cont.

\begin{tabular}{|c|c|c|c|c|c|}
\hline \multirow{2}{*}{ Ref. } & \multicolumn{3}{|c|}{ Study Details } & \multicolumn{2}{|c|}{ Observation } \\
\hline & Author (Year) & Study Design & Country/Location & Study Group & Time \\
\hline [72] & Richard et al., 2015 & $\begin{array}{l}\text { Cross-sectional, population-based study-2012 Swiss Health } \\
\text { Survey }\end{array}$ & Switzerland & $\begin{array}{c}\text { Adolescents and adults } \geq 15 \\
\text { years }\end{array}$ & 2012-2013 \\
\hline [73] & El Ansari et al., 2014 & Cross-sectional study & England, Wales, Northern Ireland & Undergraduate students & $2007-2008$ \\
\hline [74] & Mihrshahi et al., 2014 & Longitudinal study & Australia & Women & $2004,2007,2010$ \\
\hline [75] & Rutledge et al., 2014 & $\begin{array}{c}\text { Cross-sectional study - Women's Ischemia Syndrome } \\
\text { Evaluation (WISE) }\end{array}$ & USA & Women & $\begin{array}{l}\text { 1996-2000 with median of } 5.5 \\
\text { years of follow up }\end{array}$ \\
\hline [37] & Whitaker et al., 2014 & $\begin{array}{l}\text { Cross-sectional study—Sisters Taking Action for Real Success } \\
\text { (STARS) }\end{array}$ & USA/Columbia & $\begin{array}{l}\text { Overweight and obese women } \\
\text { from economically } \\
\text { disadvantaged neighborhoods }\end{array}$ & June to July $2008^{1}$ \\
\hline [76] & Akbaraly et al., 2013 & Cross-sectional study—Whitehall II & UK & Adults & $\begin{array}{l}\text { 1985-1988, 1991-1993, } \\
\text { 2003-2004, 2008-2009 }\end{array}$ \\
\hline [38] & $\begin{array}{l}\text { Bhattacharyya et al., } \\
2013\end{array}$ & Cross-sectional study & India/Goa & Adults & April 2004 to January 2005 \\
\hline [77] & McMartin et al., 2013 & $\begin{array}{l}\text { Cross-sectional, population-based study within Canadian } \\
\text { Community Health Survey (CCHS) }\end{array}$ & Canada & $\begin{array}{l}\text { Adolescents and adults } \geq 12 \\
\text { years }\end{array}$ & 2000-2009 \\
\hline [78] & Meyer et al., 2013 & $\begin{array}{c}\text { Cross-sectional, population-based study—Australian National } \\
\text { Nutrition Survey (NNS), Australian National Health Survey } \\
\text { (NHS) }\end{array}$ & Australia & Adults & 1995 \\
\hline [79] & Niu et al., 2013 & Cross-sectional, population-based study_-Tsurugaya Project & Japan/Sendai & Adults $\geq 70$ year & 2002 \\
\hline [80] & Roohafza et al., 2013 & $\begin{array}{l}\text { Cross-sectional study within Isfahan Healthy Heart Program } \\
\text { (IHHP) }\end{array}$ & Iran/Isfahan, Arak, Najafabad & Adults & Not specified \\
\hline [81] & White et al., 2013 & Micro-longitudinal study & New Zealand/Otago & Undergraduate students & April 2008 to August 2009 \\
\hline [82] & $\begin{array}{l}\text { Blanchflower et al., } \\
\qquad 2012\end{array}$ & $\begin{array}{l}\text { Cross-sectional, population-based study of the data from Welsh } \\
\text { Health Survey of 2007-2010, Scottish Health Survey of } 2008 \text { and } \\
\text { Health Survey of England in } 2008\end{array}$ & UK & Adults & $2007-2010$ \\
\hline [83] & $\begin{array}{l}\text { Davison and Kaplan } \\
2012\end{array}$ & Cross-sectional study & Canada & $\begin{array}{l}\text { Adult members of the Mood } \\
\text { Disorders Association of British } \\
\text { Columbia (MDABC) }\end{array}$ & Not specified \\
\hline [84] & Payne et al., 2012 & $\begin{array}{l}\text { Case-control study within longitudinal clinical study } \\
\text { NeuroCognitive Outcomes of Depression in the Elderly } \\
\text { (NCODE) }\end{array}$ & USA & Adults $\geq 60$ year & 1999-2007 \\
\hline [85] & Tsai et al., 2011 & $\begin{array}{l}\text { Prospective population-based study-Survey of Health and } \\
\text { Living Status of the Elderly in Taiwan (SHLSET) }\end{array}$ & Taiwan & Adults $\geq 65$ years & 1999,2003 \\
\hline [36] & Tung et al., 2011 & Cross-sectional study & Taiwan/Taipei & $\begin{array}{l}\text { Adults after coronary artery } \\
\text { bypass grafting surgery }\end{array}$ & February to June 2009 \\
\hline
\end{tabular}


Table 1. Cont

\begin{tabular}{|c|c|c|c|c|c|}
\hline \multirow{2}{*}{ Ref. } & \multicolumn{3}{|c|}{ Study Details } & \multicolumn{2}{|c|}{ Observation } \\
\hline & Author (Year) & Study Design & Country/Location & Study Group & Time \\
\hline [39] & Chai et al., 2010 & Longitudinal study & USA/Hawaii & Adults & 2-year study period \\
\hline [86] & Konttinen et al., 2010 & $\begin{array}{l}\text { Cross-sectional, population-based study-National } \\
\text { Cardiovascular Risk Factor Survey (The FINRISK } \\
\text { Study)/Dietary, Lifestyle and Genetic Determinants of Obesity } \\
\text { and Metabolic Syndrome (DILGOM substudy) }\end{array}$ & Finland & Adults & 2007 \\
\hline [87] & $\begin{array}{l}\text { Mamplekou et al., } \\
2010\end{array}$ & $\begin{array}{c}\text { Longitudinal population-based study-Mediterranean Islands } \\
\text { Elderly Study (MEDIS) }\end{array}$ & Cyprus, Greece & Adults $\geq 65$ years & $2005-2007$ \\
\hline [88] & Li et al., 2009 & $\begin{array}{l}\text { Longitudinal cross-sectional study-Third National Health and } \\
\text { Nutrition Examination Survey (NHANES III) }\end{array}$ & USA & Adults & 1988-1994 \\
\hline [89] & $\begin{array}{l}\text { Mikolajczyk et al., } \\
2009\end{array}$ & $\begin{array}{c}\text { Cross-sectional study - Cross National Student Health Survey } \\
\text { (CNSHS) }\end{array}$ & Germany, Poland, Bulgaria & First-year students & 2005 \\
\hline [90] & $\begin{array}{l}\text { Elfhag and Rasmussen } \\
2008\end{array}$ & $\begin{array}{l}\text { Cross-sectional study in Parental Influences on Their Children's } \\
\text { Health (PITCH) data set }\end{array}$ & Sweden & Women & 2000 \\
\hline [91] & Giltay et al., 2007 & Longitudinal study within Zutphen Elderly Study & Netherlands & Elderly community-living men & $1985,1990,1995,2000$ \\
\hline [92] & Liu et al., 2007 & Cross-sectional study—China Seven City Study & China & College students & $\begin{array}{l}\text { November } 2003 \text { to January } \\
2004\end{array}$ \\
\hline [93] & Kelloniemi et al., 2005 & Cross-sectional, population-based study & Finland & 31-year-old adults & $1997-1998$ \\
\hline [94] & $\begin{array}{l}\text { Sarlio-Lähteenkorva et } \\
\text { al., } 2004\end{array}$ & Cross-sectional study within Helsinki Health Study & Finland/Helsinki & Adults $40-60$ years & 2000-2001 \\
\hline [95] & Cook and Benton 1993 & Cross-sectional, population-based study & Wales/Swansea & Adults & Not specified \\
\hline
\end{tabular}

${ }^{1}$ Data provided by authors on request. 
Table 2. Characteristics of the study exposure and outcomes for the studies included to the systematic review.

\begin{tabular}{|c|c|c|c|c|c|}
\hline \multirow[b]{2}{*}{ Ref. } & \multicolumn{3}{|c|}{ Exposure } & \multicolumn{2}{|r|}{ Outcomes } \\
\hline & Assessment & $\begin{array}{c}\text { Measure of Fruit and } \\
\text { Vegetable }\end{array}$ & Other Fruit/Vegetable Products & Assessment & Psychological Measure \\
\hline [40] & Rapid Food Screener & $\begin{array}{l}\text { Frequency of } \\
\text { consumption }\end{array}$ & $\begin{array}{c}\text { Fruits including juices } \\
\text { Vegetables including salads, soups }\end{array}$ & Stress & Perceived Stress Scale \\
\hline [41] & $\begin{array}{l}\text { Semi-quantitative food frequency } \\
\text { questionnaire }\end{array}$ & $\begin{array}{l}\text { Portion size, frequency of } \\
\text { consumption }\end{array}$ & Not specified & Depressive symptoms & Center for Epidemiological Studies Depression Scale (CESD-10) \\
\hline [42] & $\begin{array}{l}\text { Question about fruit and } \\
\text { vegetable consumption }\end{array}$ & $\begin{array}{l}\text { Frequency of } \\
\text { consumption }\end{array}$ & Not specified & $\begin{array}{l}\text { (1) Depression } \\
\text { (2) Quality of life }\end{array}$ & $\begin{array}{l}\text { (1) EURO-D scale (depression scale) } \\
\text { (2) CASP-12 with subscales for Control, Autonomy, Self-realization } \\
\text { and Pleasure }\end{array}$ \\
\hline [43] & $\begin{array}{l}\text { Question about fruit and } \\
\text { vegetable consumption on a } \\
\text { typical day with a list of } \\
\text { country-specific examples of } \\
\text { fruits and vegetables }\end{array}$ & $\begin{array}{l}\text { Frequency of } \\
\text { consumption }\end{array}$ & Not specified & $\begin{array}{l}\text { (1) Depressive symptoms } \\
\text { (2) Quality of life }\end{array}$ & $\begin{array}{l}\text { (1) Number of depressive symptoms during the previous } 2 \text { weeks } \\
\text { (loss of appetite, slowed-down thinking, problems falling asleep, } \\
\text { waking up too early, difficulties concentrating, slowing down } \\
\text { in moving around, feeling anxious or worried, being restless, } \\
\text { feeling negative about oneself, feeling hopeless, losing interest } \\
\text { in sex, suicidal ideation, suicidal behavior) } \\
\text { (2) 8-items version of the WHO Quality of Life scale (WHOQOL) }\end{array}$ \\
\hline [44] & $\begin{array}{l}\text { Question about fruit and } \\
\text { vegetable consumption over the } \\
\text { last } 3 \text { days }\end{array}$ & $\begin{array}{l}\text { Frequency of } \\
\text { consumption }\end{array}$ & Vegetables including salads & $\begin{array}{c}\text { Depression and } \\
\text { subsyndromal depression }\end{array}$ & Geriatric Mental State (GMS) \\
\hline [45] & $\begin{array}{l}\text { Questions about fruit and } \\
\text { vegetable consumption on a } \\
\text { typical day when they are } \\
\text { consumed, fruit and vegetable } \\
\text { consumption on a typical week }\end{array}$ & $\begin{array}{l}\text { Portion size, frequency of } \\
\text { consumption }\end{array}$ & Not specified & Mental well-being & 12-item General Health Questionnaire (GHQ-12) \\
\hline [35] & WHO STEPS Instrument & $\begin{array}{l}\text { Frequency of } \\
\text { consumption }\end{array}$ & Not specified & $\begin{array}{l}\text { (1) Mental components } \\
\text { of health } \\
\text { (2) Major depression } \\
\text { (3) Anxiety }\end{array}$ & $\begin{array}{l}\text { (1) Short Form (SF-8) Health Survey } \\
\text { (2) Patient Health Questionnaire-9 (PHQ-9) } \\
\text { (3) Generalized Anxiety Disorder (GAD-7) }\end{array}$ \\
\hline [46] & $\begin{array}{l}\text { Food frequency questionnaire } \\
\text { with } 55 \text { food items }\end{array}$ & $\begin{array}{c}\text { Frequency of } \\
\text { consumption recalculated } \\
\text { into Mediterranean Diet } \\
\text { Serving Score (MDSS) } \\
\end{array}$ & Not specified & $\begin{array}{l}\text { Minor psychiatric disorders } \\
\text { and psychological distress }\end{array}$ & General Health Questionnaire-30 (GHQ-30) \\
\hline [47] & $\begin{array}{l}\text { Semi-quantitative food frequency } \\
\text { questionnaire with } 27 \text { food items }\end{array}$ & $\begin{array}{l}\text { Frequency of } \\
\text { consumption }\end{array}$ & Not specified & $\begin{array}{l}\text { Health-related quality of life } \\
\text { (HR-QoL) }\end{array}$ & $\begin{array}{l}\text { Short Form 36-Health Survey (SF-36), including Mental Health } \\
\text { Component (MH) }\end{array}$ \\
\hline
\end{tabular}


Table 2. Cont.

\begin{tabular}{|c|c|c|c|c|c|}
\hline \multirow[b]{2}{*}{ Ref. } & \multicolumn{3}{|c|}{ Exposure } & \multicolumn{2}{|r|}{ Outcomes } \\
\hline & Assessment & $\begin{array}{l}\text { Measure of Fruit and } \\
\text { Vegetable }\end{array}$ & Other Fruit/Vegetable Products & Assessment & Psychological Measure \\
\hline [48] & $\begin{array}{l}\text { Semi-quantitative food frequency } \\
\text { questionnaire with } 147 \text { food items } \\
\text { and } 14 \text { items related to local } \\
\text { spices and vegetables }\end{array}$ & $\begin{array}{l}\text { Frequency of } \\
\text { consumption converted to } \\
\text { g/day }\end{array}$ & Fruits including juices & Depression, anxiety and stress & The Depression, Anxiety, Stress Scales (DASS, 21-items) \\
\hline [49] & $\begin{array}{l}\text { Questions about fruit and } \\
\text { vegetable consumption on a } \\
\text { previous day }\end{array}$ & $\begin{array}{l}\text { Frequency of } \\
\text { consumption }\end{array}$ & $\begin{array}{l}\text { Fruits including canned, dried, } \\
\text { fruit juices } \\
\text { Vegetables including salads }\end{array}$ & Psychological well-being & $\begin{array}{l}\text { 17-items from the Control, Autonomy, Satisfaction, Pleasure Scale } \\
\text { (CASP-17) }\end{array}$ \\
\hline [50] & $\begin{array}{l}\text { Questions about number of days } \\
\text { a week when fruit and vegetables } \\
\text { are consumed, and consumption } \\
\text { on a typical day when they are } \\
\text { consumed }\end{array}$ & $\begin{array}{l}\text { Frequency of } \\
\text { consumption for raw and } \\
\text { processed ones }\end{array}$ & $\begin{array}{l}\text { Fruit including canned } \\
\text { Vegetables including canned }\end{array}$ & $\begin{array}{ll}\text { (1) } & \text { Depressive symptoms } \\
\text { (2) } & \text { Anxiety } \\
\text { (3) } & \text { Negative } \\
& \text { positive mood and } \\
\text { (4) } & \text { Life satisfaction } \\
\text { (5) } & \text { Flourishing }\end{array}$ & $\begin{array}{l}\text { (1) Centre for Epidemiological Depression Scale (CESD) } \\
\text { (2) 7-items Hospital Anxiety and Depression Scale-Anxiety } \\
\text { Subscale (HADS-A) } \\
\text { (3) Question about } 12 \text { negative (hostile, stressed, irritable, angry, } \\
\text { anxious, annoyed, nervous, tense, hopeless, unhappy, dejected, } \\
\text { sad) and } 12 \text { positive affects (enthusiastic, excited, energetic, } \\
\text { joyful, happy, cheerful, pleasant, good, relaxed, calm, } \\
\text { content, satisfied) } \\
\text { (4) Satisfaction with Life Scale (SWLS) } \\
\text { (5) Flourishing Scale }\end{array}$ \\
\hline [51] & $\begin{array}{l}\text { Question about fruit and } \\
\text { vegetable consumption on a } \\
\text { previous day }\end{array}$ & $\begin{array}{l}\text { Frequency of } \\
\text { consumption }\end{array}$ & $\begin{array}{l}\text { Fruits including fruit juices } \\
\text { Vegetables including vegetable } \\
\text { juices }\end{array}$ & Depressive symptoms & Center for Epidemiological Studies Depression Scale (CESD-10) \\
\hline [52] & $\begin{array}{l}\text { Nutritional questionnaire to } \\
\text { assess intake of products for } \\
\text { Mediterranean Diet adherence } \\
\text { score (MeDi) and Index of Diet } \\
\text { Quality (IDQ) }\end{array}$ & $\begin{array}{l}\text { Frequency of } \\
\text { consumption }\end{array}$ & $\begin{array}{l}100 \% \text { juice as a separate group } \\
\text { Vegetables including salads }\end{array}$ & Perceived happiness & Visual Analog Scale of Happiness (0-100 mm) \\
\hline [53] & Not specified & $\begin{array}{l}\text { Frequency of } \\
\text { consumption recalculated } \\
\text { into Mediterranean Diet } \\
\text { Score } \\
\end{array}$ & Not specified & Depressive symptoms & Geriatric Depression Scale (GDS) \\
\hline [54] & $\begin{array}{l}\text { Semi-quantitative food frequency } \\
\text { questionnaire with } 106 \text { food items }\end{array}$ & $\begin{array}{l}\text { Total fruit and total } \\
\text { vegetables intake }\end{array}$ & $\begin{array}{c}\text { Fruit including juices, dried and } \\
\text { herbs } \\
\text { Vegetables including salads }\end{array}$ & $\begin{array}{l}\text { (1) Anxiety and depression } \\
\text { (2) Psychological distress }\end{array}$ & $\begin{array}{l}\text { (1) Iranian-validated version of Hospital Anxiety and Depression } \\
\text { Scale (HADS) } \\
\text { (2) Iranian-validated version of General Health Questionnaire } \\
\text { (GHQ) }\end{array}$ \\
\hline
\end{tabular}


Table 2. Cont

\begin{tabular}{|c|c|c|c|c|c|}
\hline \multirow[b]{2}{*}{ Ref. } & \multicolumn{3}{|c|}{ Exposure } & \multicolumn{2}{|r|}{ Outcomes } \\
\hline & Assessment & $\begin{array}{l}\text { Measure of Fruit and } \\
\text { Vegetable }\end{array}$ & Other Fruit/Vegetable Products & Assessment & Psychological Measure \\
\hline [55] & $\begin{array}{l}\text { Question about eating } 5 \text { portions } \\
\text { of fruit and vegetables daily } \\
\text { during last weeks }\end{array}$ & $\begin{array}{l}\text { 5-point Likert scale for } \\
\text { following } \\
\text { recommendation }\end{array}$ & Not specified & $\begin{array}{l}\text { (1) Restful sleep; } \\
\text { (2) Sleep quality; } \\
\text { (3) Quality of life and } \\
\text { subjective health }\end{array}$ & $\begin{array}{l}\text { (1) Center for Epidemiological Studies Depression Scale (CESD-10) } \\
\text { item-level of restful sleep; } \\
\text { (2) World Health Organization Quality of Life (WHOQOL-BREF) } \\
\text { Questionnaire question-level of satisfaction with sleep; } \\
\text { (3) WHOQOL-BREF Questionnaire question—level of quality of life }\end{array}$ \\
\hline [56] & $\begin{array}{l}\text { Two items from the National } \\
\text { Cancer Institute Quick Food Scan }\end{array}$ & $\begin{array}{l}\text { Frequency of } \\
\text { consumption }\end{array}$ & $\begin{array}{l}\text { Fruit including } 100 \% \text { pure juices } \\
\text { Vegetables including } 100 \% \text { pure } \\
\text { juices }\end{array}$ & Health-Related Self-Efficacy & $\begin{array}{l}\text { Question: "Overall, how confident are you about your ability to take } \\
\text { good care of your health?" using a 5-point Likert scale ranging from } \\
\text { (1) completely confident to (5) not at all confident }\end{array}$ \\
\hline [57] & $\begin{array}{l}\text { Question about fruit } \\
\text { consumption on a typical day }\end{array}$ & $\begin{array}{l}\text { Frequency of } \\
\text { consumption }\end{array}$ & Not specified & $\begin{array}{l}\text { Self-reported depression } \\
\text { status }\end{array}$ & $\begin{array}{l}\text { (1) question: "During the last } 12 \text { months, have you had a period } \\
\text { lasting several days when you felt sad, empty or depressed?" } \\
\text { Yes/No } \\
\text { (2) question: "Overall in the last } 30 \text { days, how much of a } \\
\text { problem did you have with feeling sad, low or depressed?" } \\
\text { None/Mild/Moderate/Severe/Extreme }\end{array}$ \\
\hline [58] & $\begin{array}{l}\text { Validated questions about fruit } \\
\text { and vegetable consumption on a } \\
\text { typical day }\end{array}$ & $\begin{array}{l}\text { Frequency of } \\
\text { consumption }\end{array}$ & $\begin{array}{l}\text { Fruits including canned } \\
\text { Vegetables including salads }\end{array}$ & Psychological distress & 10-items Kessler Psychological Distress Scale (K10) \\
\hline [59] & $\begin{array}{l}\text { Question about fruit and } \\
\text { vegetable consumption on a } \\
\text { typical day }\end{array}$ & $\begin{array}{l}\text { Frequency of } \\
\text { consumption }\end{array}$ & Not specified & $\begin{array}{l}\text { (1) Happiness } \\
\text { (2) Depressive symptoms }\end{array}$ & $\begin{array}{l}\text { (1) Happiness Scale } \\
\text { (2) Center for Epidemiological Studies Depression Scale (CESD-10) }\end{array}$ \\
\hline [60] & $\begin{array}{l}\text { Question about fruit and } \\
\text { vegetable consumption on a } \\
\text { typical day }\end{array}$ & $\begin{array}{l}\text { Frequency of } \\
\text { consumption, intake }\end{array}$ & Fruit including juices & $\begin{array}{l}\text { Clinically-relevant levels of } \\
\text { depressive symptoms }\end{array}$ & Center for Epidemiological Studies Depression Scale (CES-D) \\
\hline [61] & $\begin{array}{l}\text { Either semi-quantitative food } \\
\text { frequency questionnaire with } 90 \\
\text { food items (CoLaus/PsyCoLauS), } \\
\text { or question about fruit and } \\
\text { vegetable consumption on a } \\
\text { typical day (SHS) }\end{array}$ & $\begin{array}{l}\text { Frequency of } \\
\text { consumption }\end{array}$ & $\begin{array}{l}\text { Fruits including juices } \\
\text { Vegetables including salads and } \\
\text { juices }\end{array}$ & Depression & $\begin{array}{c}\text { Either semi-structured Diagnostic Interview for Genetic Studies } \\
\text { (DIGS) (CoLaus/PsyCoLauS), or Patient Health Questionnaire } \\
\text { (PHQ-9) (SHS) }\end{array}$ \\
\hline [62] & $\begin{array}{l}\text { The National Cancer Institute } \\
\text { (NCI) All Day Fruit and } \\
\text { Vegetable Screener }\end{array}$ & $\begin{array}{l}\text { Portion size, frequency of } \\
\text { consumption }\end{array}$ & $\begin{array}{l}\text { Vegetables including salads and } \\
\text { vegetable soups }\end{array}$ & $\begin{array}{l}\text { (1) Well-being } \\
\text { (2) Positive affect and } \\
\text { negative affect }\end{array}$ & $\begin{array}{l}\text { (1) Satisfaction with Life Scale (LS) } \\
\text { (2) The Positive and Negative Affect Schedule (PANAS) }\end{array}$ \\
\hline
\end{tabular}


Table 2. Cont.

\begin{tabular}{|c|c|c|c|c|c|}
\hline \multirow[b]{2}{*}{ Ref. } & \multicolumn{3}{|c|}{ Exposure } & \multicolumn{2}{|r|}{ Outcomes } \\
\hline & Assessment & $\begin{array}{l}\text { Measure of Fruit and } \\
\text { Vegetable }\end{array}$ & Other Fruit/Vegetable Products & Assessment & Psychological Measure \\
\hline [63] & WHO STEPS Instrument & Number of portions & Not specified & $\begin{array}{l}\text { Depressive symptoms } \\
\text { (including major depressive } \\
\text { syndrome) }\end{array}$ & Patient Health Questionnaire (PHQ-9) \\
\hline [64] & $\begin{array}{l}\text { Validated semi-quantitative } \\
\text { questionnaire }\end{array}$ & $\begin{array}{l}\text { Frequency of } \\
\text { consumption }\end{array}$ & Not specified & Depressive symptoms & Center for Epidemiological Studies Depression Scale (CESD-10) \\
\hline [65] & $\begin{array}{l}\text { Questions about number of days } \\
\text { a week when fruit and vegetables } \\
\text { are consumed, and consumption } \\
\text { on a typical day when they are } \\
\text { consumed }\end{array}$ & $\begin{array}{l}\text { Frequency of } \\
\text { consumption }\end{array}$ & $\begin{array}{l}\text { Fruit including canned and juices } \\
\text { Vegetables including canned and } \\
\text { juices }\end{array}$ & Happiness score & Oxford Happiness Questionnaire (OHQ) \\
\hline [66] & $\begin{array}{l}\text { Questions about number of days } \\
\text { a week when fruit and vegetables } \\
\text { are consumed, and consumption } \\
\text { on a typical day when they are } \\
\text { consumed }\end{array}$ & $\begin{array}{l}\text { Frequency of } \\
\text { consumption }\end{array}$ & $\begin{array}{l}\text { Fruit including canned and dried } \\
\text { Vegetables including canned }\end{array}$ & Self-reported life satisfaction & $\begin{array}{l}\text { (1) Question about subjective assessment how satisfied with his } \\
\text { life is respondent } \\
\text { (2) Medical Outcomes Short Form (SF-36)—question about being a } \\
\text { happy person }\end{array}$ \\
\hline [67] & $\begin{array}{c}\text { Question about fruit and } \\
\text { vegetable included to diet at least } \\
\text { monthly; } \\
\text { Question about fruit and } \\
\text { vegetable consumption on a } \\
\text { typical day }\end{array}$ & $\begin{array}{l}\text { Frequency of } \\
\text { consumption }\end{array}$ & Not specified & Depression, anxiety and stress & The Depression, Anxiety, Stress Scales (DASS, 21-items) \\
\hline [68] & $\begin{array}{l}\text { Question about fruit and } \\
\text { vegetable consumption on a } \\
\text { present day }\end{array}$ & $\begin{array}{l}\text { Frequency of } \\
\text { consumption }\end{array}$ & $\begin{array}{l}\text { Juices, dried fruits and vegetables } \\
\text { excluded }\end{array}$ & $\begin{array}{ll}\text { (1) } & \text { Daily } \\
\text { eudaemonic well-being } \\
\text { (2) } & \text { Daily curiosity } \\
\text { (3) } & \text { Daily creativity } \\
\text { (4) } & \text { Daily affect }\end{array}$ & $\begin{array}{l}\text { (1) Adaptation of the Flourishing Scale (8-items) } \\
\text { (2) Curiosity and Exploration Inventory (7-items) } \\
\text { (3) Question about subjective assessment of own creativity level } \\
\text { on a present day } \\
\text { (4) Question about subjective assessment of own affects on a present } \\
\text { day-9 items for positive affects (calm, content, relaxed, happy, } \\
\text { cheerful, pleasant, energetic, enthusiastic, and excited) and 9 } \\
\text { for negative affcts (sad, dejected, depressed, nervous, anxious, } \\
\text { tense, angry, irritable, and hostile }\end{array}$ \\
\hline [69] & $\begin{array}{l}\text { Question about fruit and } \\
\text { vegetable consumption on a } \\
\text { typical day }\end{array}$ & $\begin{array}{l}\text { Frequency of } \\
\text { consumption }\end{array}$ & Juices excluded & $\begin{array}{l}\text { (1) Major depression } \\
\text { (2) Psychological distress }\end{array}$ & $\begin{array}{l}\text { (1) Composite International Diagnostic Interview Short Form } \\
\text { (CIDI-SF) } \\
\text { (2) Kessler Psychological Distress Scale (K6) }\end{array}$ \\
\hline
\end{tabular}


Table 2. Cont.

\begin{tabular}{|c|c|c|c|c|c|}
\hline \multirow[b]{2}{*}{ Ref. } & \multicolumn{3}{|c|}{ Exposure } & \multicolumn{2}{|r|}{ Outcomes } \\
\hline & Assessment & $\begin{array}{l}\text { Measure of Fruit and } \\
\text { Vegetable }\end{array}$ & Other Fruit/Vegetable Products & Assessment & Psychological Measure \\
\hline [70] & 6-items food frequency screener & $\begin{array}{l}\text { Frequency of } \\
\text { consumption }\end{array}$ & $\begin{array}{l}\text { Fruit juices and green salad } \\
\text { included in the screener as a } \\
\text { separate question }\end{array}$ & Quality of life & $\begin{array}{l}\text { Health-related quality of life (HRQOL) (including self-reported } \\
\text { mental health, days of poor mental health in the past month, days of } \\
\text { limited activities because of poor physical/mental health in the past } \\
\text { month) }\end{array}$ \\
\hline [71] & $\begin{array}{l}\text { Commonwealth Scientific and } \\
\text { Industrial Research Organization } \\
\text { food frequency questionnaire } \\
\text { (CSIRO FFQ) }\end{array}$ & $\begin{array}{l}\text { Frequency of } \\
\text { consumption }\end{array}$ & Fruit/vegetables excluding juices & Stress & Depression Anxiety Stress Scale (DASS)—Stress subscale \\
\hline [72] & $\begin{array}{l}\text { Food frequency questionnaire } \\
\text { including questions about } \\
\text { number of days a week when } \\
\text { fruit and vegetables are } \\
\text { consumed, and consumption on a } \\
\text { typical day when they are } \\
\text { consumed }\end{array}$ & $\begin{array}{l}\text { Frequency of } \\
\text { consumption, following } \\
\text { recommendations }\end{array}$ & $\begin{array}{l}\text { Fruits including juices } \\
\text { Vegetables including salads and } \\
\text { juices }\end{array}$ & Psychological distress & 5-items Mental Health Inventory (MHI-5) \\
\hline [73] & Food frequency questionnaire & $\begin{array}{l}\text { Frequency of } \\
\text { consumption }\end{array}$ & Vegetables including salads & $\begin{array}{l}\text { Stress } \\
\text { Depressive symptoms }\end{array}$ & $\begin{array}{l}\text { (1) Cohen's Perceived Stress Scale (PSS-4 items) } \\
\text { (2) Modification of the Beck Depression Inventory (MBDI) }\end{array}$ \\
\hline [74] & $\begin{array}{l}\text { Question about fruit and } \\
\text { vegetable consumption on a } \\
\text { typical day }\end{array}$ & $\begin{array}{l}\text { Frequency of } \\
\text { consumption }\end{array}$ & $\begin{array}{l}\text { Vegetables including salads and } \\
\text { potatoes }\end{array}$ & Depressive symptoms & Center for Epidemiological Studies Depression Scale (CESD-10) \\
\hline [75] & $\begin{array}{l}1998 \text { Block Food Frequency } \\
\text { Questionnaire for Adults }\end{array}$ & $\begin{array}{l}\text { Frequency of } \\
\text { consumption }\end{array}$ & Not specified & Depression & $\begin{array}{l}\text { The Beck Depression Inventory (21-items); } \\
\text { Current use of antidepressants; } \\
\text { Self-reported history of treatment for depression }\end{array}$ \\
\hline [37] & Three 24-h dietary recalls & $\begin{array}{l}\text { Frequency of } \\
\text { consumption }\end{array}$ & $\begin{array}{l}\text { Fruits including canned, dried, } \\
\text { juices } \\
\text { Vegetables including juices }\end{array}$ & Depressive symptoms & Center for Epidemiological Studies Depression Scale (CESD-10) \\
\hline [76] & $\begin{array}{l}\text { Semi-quantitative food-frequency } \\
\text { questionnaire with } 127 \text { food items }\end{array}$ & $\begin{array}{l}\text { Intake recalculated into } \\
\text { the Alternative Healthy } \\
\text { Eating Index (AHEI), } \\
\text { including among others } \\
\text { component of fruits and } \\
\text { of vegetables }\end{array}$ & Not specified & Depressive symptoms & $\begin{array}{l}\text { Center for Epidemiological Studies Depression Scale (CESD-10); } \\
\text { Self-reported use of antidepressants }\end{array}$ \\
\hline [38] & $\begin{array}{l}\text { Food frequency questionnaire } \\
\text { with } 63 \text { food items }\end{array}$ & $\begin{array}{l}\text { Frequency of } \\
\text { consumption }\end{array}$ & Fruit including canned & Psychological distress & Kessler Psychological Distress Scale (K10) \\
\hline
\end{tabular}


Table 2. Cont

\begin{tabular}{|c|c|c|c|c|c|}
\hline \multirow[b]{2}{*}{ Ref. } & \multicolumn{3}{|c|}{ Exposure } & \multicolumn{2}{|r|}{ Outcomes } \\
\hline & Assessment & $\begin{array}{c}\text { Measure of Fruit and } \\
\text { Vegetable }\end{array}$ & Other Fruit/Vegetable Products & Assessment & Psychological Measure \\
\hline [77] & $\begin{array}{l}\text { Question about fruit and } \\
\text { vegetable consumption on a } \\
\text { typical day }\end{array}$ & $\begin{array}{l}\text { Frequency of } \\
\text { consumption }\end{array}$ & Juices excluded & $\begin{array}{l}\text { Major } \\
\text { depressive episode } \\
\text { Psychological distress } \\
\text { Self-perceived } \\
\text { mental health } \\
\begin{array}{l}\text { Physician-diagnosed } \\
\text { mood and } \\
\text { anxiety disorder }\end{array}\end{array}$ & $\begin{array}{l}\text { (1) Composite International Diagnostic Interview-Short Form } \\
\text { (CIDI-SF) } \\
\text { (2) Kessler Psychological Distress Scale (K6) } \\
\text { (3) Question about self-perceived mental health } \\
\text { (4) Question about chronic conditions diagnosed by a health } \\
\text { professional for a mood disorder, such as (A) depression, bipolar } \\
\text { disorder, mania or dysthymia, (B) anxiety disorder such as a } \\
\text { phobia, obsessive-compulsive disorder or a panic disorder }\end{array}$ \\
\hline [78] & $\begin{array}{l}\text { 24-h dietary recall; } \\
\text { Question about frequency of } \\
\text { consumption }\end{array}$ & $\begin{array}{l}\text { Number of portions, } \\
\text { frequency of consumption }\end{array}$ & Not specified & Depression & $\begin{array}{l}\text { Question about any recent illness (medical conditions during } \\
\text { previous } 2 \text { weeks) or long-term illness (lasted at least } 6 \text { months), } \\
\text { screened for depression, based on ICD-10 }\end{array}$ \\
\hline [79] & $\begin{array}{l}\text { Brief self-administered Diet } \\
\text { History Questionnaire (BDHQ) } \\
\text { with } 75 \text { food items }\end{array}$ & $\begin{array}{l}\text { Frequency of } \\
\text { consumption }\end{array}$ & $\begin{array}{l}\text { Tomato products including tomato } \\
\text { ketchup, stewed tomato, or tomato } \\
\text { stew }\end{array}$ & Depressive symptoms & 30-items Japanese version of Geriatric Depression Scale (GDS) \\
\hline [80] & $\begin{array}{l}\text { Food frequency questionnaire } \\
\text { with } 49 \text { food items }\end{array}$ & $\begin{array}{l}\text { Frequency of } \\
\text { consumption }\end{array}$ & Not specified & Psychologic stress & General Health Questionnaire-12 (GHQ-12) \\
\hline [81] & $\begin{array}{l}\text { Question about fruit and } \\
\text { vegetable consumption on a } \\
\text { typical day }\end{array}$ & $\begin{array}{l}\text { Frequency of } \\
\text { consumption }\end{array}$ & Juices and dried fruits excluded & Negative and positive affects & $\begin{array}{l}\text { Questions about } 9 \text { negative (depressed, sad, unhappy, anxious, } \\
\text { nervous, tense, angry, hostile, short-tempered) and } 9 \text { positive affects } \\
\text { (calm, content, relaxed, cheerful, happy, pleased, energetic, } \\
\text { enthusiastic, excited) }\end{array}$ \\
\hline [82] & Not specified & $\begin{array}{l}\text { Frequency of } \\
\text { consumption }\end{array}$ & Fruit including orange juice & $\begin{array}{l}\text { Depending on the study: } \\
\text { (1) Life satisfaction } \\
\text { (2) Well-being } \\
\text { (3) Mental } \\
\text { ill-being/mental distress } \\
\text { (4) Happiness } \\
\text { (5) Nervousness } \\
\text { (6) Being downhearted } \\
\text { and low }\end{array}$ & $\begin{array}{l}\text { Depending on the study: } \\
\text { (1) Question about self-reported life satisfaction } \\
\text { (2) The Warwick-Edinburgh Mental Wellbeing Scale (WEMWBS) } \\
\text { (3) General Health Questionnaire GHQ (including mental } \\
\text { ill-being/mental distress questions) } \\
\text { (4) Question about self-reported being happy } \\
\text { (5) Question about self-reported being nervous } \\
\text { (6) Question about self-reported being downhearted and low }\end{array}$ \\
\hline
\end{tabular}


Table 2. Cont.

\begin{tabular}{|c|c|c|c|c|c|}
\hline \multirow[b]{2}{*}{ Ref. } & \multicolumn{3}{|c|}{ Exposure } & \multicolumn{2}{|r|}{ Outcomes } \\
\hline & Assessment & $\begin{array}{l}\text { Measure of Fruit and } \\
\text { Vegetable }\end{array}$ & Other Fruit/Vegetable Products & Assessment & Psychological Measure \\
\hline [83] & $\begin{array}{l}\text { 3-day dietary record; } \\
\text { Validated food frequency } \\
\text { questionnaire }\end{array}$ & $\begin{array}{l}\text { Frequency of } \\
\text { consumption }\end{array}$ & Fruits including juices and nectars & Depression & $\begin{array}{c}\text { Structured Clinical Interview for DSM-IV Axis I Disorders, Global } \\
\text { Assessment of Functioning (GAF) Scale, Hamilton Depression Scale } \\
\text { (Ham-D), Young Mania Rating Scale (YMRS) }\end{array}$ \\
\hline [84] & $\begin{array}{l}1998 \text { Block Food Frequency } \\
\text { Questionnaire }\end{array}$ & $\begin{array}{l}\text { Frequency of } \\
\text { consumption }\end{array}$ & Not specified & $\begin{array}{l}\text { Depression; } \\
\text { Self-reported physical health }\end{array}$ & $\begin{array}{c}\text { Duke Depression Evaluation Schedule (sections of the National } \\
\text { Institute of Mental Health (NIMH) Diagnostic Interview Schedule } \\
\text { which assesses depression, as well as items on self-reported physical } \\
\text { health) }\end{array}$ \\
\hline [85] & $\begin{array}{l}\text { Question about fruit and } \\
\text { vegetable consumption on a } \\
\text { typical week }\end{array}$ & $\begin{array}{l}\text { Frequency of } \\
\text { consumption }\end{array}$ & Not specified & $\begin{array}{l}\text { Depressive symptoms } \\
\text { (interpreted as having a risk } \\
\text { of depression) }\end{array}$ & Center for Epidemiological Studies Depression Scale (CESD-10) \\
\hline [36] & $\begin{array}{l}\text { Chinese Food Frequency } \\
\text { Questionnaire-Short Form (Short } \\
\text { C-FFQ) }\end{array}$ & $\begin{array}{l}\text { Frequency of } \\
\text { consumption }\end{array}$ & Not specified & Quality of life & Taiwanese version of the Short Form 36-Health Survey (SF-36) \\
\hline [39] & NCI Fruit and Vegetable screener & $\begin{array}{l}\text { Frequency of } \\
\text { consumption of } 9 \\
\text { categories of fruits and } \\
\text { vegetables }\end{array}$ & $\begin{array}{l}\text { Fruit and vegetables (including } \\
\text { fruit, fruit juices, salad, beans, } \\
\text { French fries, other potatoes, tomato } \\
\text { sauce, vegetable soups and other } \\
\text { vegetables) }\end{array}$ & Quality of life & SF-12 Health Survey \\
\hline [86] & $\begin{array}{l}\text { 132-item food frequency } \\
\text { questionnaire }\end{array}$ & $\begin{array}{l}\text { Frequency of } \\
\text { consumption }\end{array}$ & $\begin{array}{c}\text { Mayonnaise salads excluded from } \\
\text { vegetables }\end{array}$ & Depressive symptoms & Center for Epidemiological Studies Depression Scale (CESD-10) \\
\hline [87] & $\begin{array}{l}\text { Semi-quantitative food-frequency } \\
\text { questionnaire }\end{array}$ & $\begin{array}{l}\text { Frequency of } \\
\text { consumption }\end{array}$ & Not specified & Depressive symptoms & Shortened version of Geriatric Depression Scale (GDS) \\
\hline [88] & $\begin{array}{l}\text { Qualitative } 60 \text {-items food } \\
\text { frequency questionnaire; } 24-\mathrm{h} \\
\text { dietary recall }\end{array}$ & $\begin{array}{l}\text { Frequency of } \\
\text { consumption }\end{array}$ & Fruits including juices & Attempted suicide & $\begin{array}{l}\text { Simple question ("Have you ever attempted suicide?"), according to } \\
\text { the standardized criteria established in the Diagnostic Interview } \\
\text { Schedule }\end{array}$ \\
\hline [89] & Food frequency questionnaire & $\begin{array}{l}\text { Frequency of } \\
\text { consumption }\end{array}$ & Vegetables including salads & $\begin{array}{l}\text { Perceived stress } \\
\text { Depressive symptoms }\end{array}$ & $\begin{array}{l}\text { (1) Cohen's Perceived Stress Scale (PSS-14 items) } \\
\text { (2) Modification of Beck Depression Inventory (M-BDI) }\end{array}$ \\
\hline [90] & Food frequency questionnaire & $\begin{array}{l}\text { Frequency of } \\
\text { consumption }\end{array}$ & Not specified & Self-esteem & Harter Self-Perception Profile for Adults (SPPA) \\
\hline
\end{tabular}


Table 2. Cont

\begin{tabular}{|c|c|c|c|c|c|}
\hline \multirow[b]{2}{*}{ Ref. } & \multicolumn{3}{|c|}{ Exposure } & \multicolumn{2}{|r|}{ Outcomes } \\
\hline & Assessment & $\begin{array}{l}\text { Measure of Fruit and } \\
\text { Vegetable }\end{array}$ & Other Fruit/Vegetable Products & Assessment & Psychological Measure \\
\hline [91] & $\begin{array}{l}\text { Cross-check dietary history } \\
\text { method }\end{array}$ & $\begin{array}{l}\text { Frequency of } \\
\text { consumption, intake }\end{array}$ & Not specified & Dispositional optimism & $\begin{array}{l}\text { 4-item questionnaire-"I still expect much from life," "I do not look } \\
\text { forward to what lies ahead for me in the years to come," "My days } \\
\text { seem to be passing by slowly," and "I am still full of plans" }\end{array}$ \\
\hline [92] & Food frequency questionnaire & $\begin{array}{l}\text { Frequency of } \\
\text { consumption }\end{array}$ & Fruits including juices & $\begin{array}{ll}\text { (1) } & \text { Stress } \\
\text { (2) } & \text { Depression }\end{array}$ & $\begin{array}{l}\text { (1) Perceived Stress Scale (PSS) } \\
\text { (2) Center for Epidemiological Studies Depression Scale (CES-D) }\end{array}$ \\
\hline [93] & $\begin{array}{l}\text { Food frequency questionnaire } \\
\text { with } 32 \text { food items }\end{array}$ & $\begin{array}{l}\text { Frequency of } \\
\text { consumption }\end{array}$ & Vegetables including salads & Dispositional optimism & Life Orientation Test (LOT-R) \\
\hline [94] & Food frequency inventory & $\begin{array}{l}\text { Frequency of } \\
\text { consumption }\end{array}$ & $\begin{array}{l}\text { Fruits including juices } \\
\text { Vegetables including salads }\end{array}$ & Mental health status & $\begin{array}{l}\text { (1) General Health Questionnaire (GHQ-12) } \\
\text { (2) History of lifetime mental diagnosis (for depression, anxiety, } \\
\text { eating disorder, any other mental health problem) }\end{array}$ \\
\hline [95] & Food frequency questionnaire & $\begin{array}{l}\text { Frequency of } \\
\text { consumption }\end{array}$ & $\begin{array}{c}\text { Fruits including dried, canned, } \\
\text { pure juices } \\
\text { Vegetables including salads, } \\
\text { potatoes (not chips) }\end{array}$ & Anxiety, depression & General Health Questionnaire-30 (GHQ-30) \\
\hline
\end{tabular}


Table 3. Characteristics of the study findings for the studies included to the systematic review accompanied by the quality assessment based on the total score for the Newcastle-Ottawa Scale.

\begin{tabular}{|c|c|c|c|}
\hline \multirow{2}{*}{ Ref. } & \multicolumn{2}{|l|}{ Findings } & \multirow{2}{*}{ Quality * } \\
\hline & Observation & Conclusion & \\
\hline [40] & $\begin{array}{l}\text { Women with high stress consumed significantly less fruits }(p=0.01) \text { and vegetables }(p=0.02) \\
\text { than women with low stress, with effect sizes of } d=0.24 \text { and } 0.25 \text {, respectively, for the } \\
\text { between-group differences. }\end{array}$ & $\begin{array}{l}\text { Nutrition counselling on increasing fruit and vegetable intakes may consider targeting } \\
\text { women who are black or younger or who report high stress, respectively. }\end{array}$ & 5 \\
\hline [41] & $\begin{array}{l}\text { After adjustment for confounding variables, participants in the highest quartile of the fruits } \\
\text { consumption and vegetables consumption had lower prevalence ratio (PR) for depressive } \\
\text { symptoms (PR }=0.76 ; 95 \% \text { confidence interval (CI): } 0.603-0.974, p=0.042 ; \mathrm{PR}=0.77 ; 95 \% \text { CI: } \\
0.612-0.977, p=0.045 \text { ) than those in the lowest quartile. Those in the highest quartile of total } \\
\text { vegetables and fruits consumption had also a lower PR of depressive symptoms (PR }=0.67 ; 95 \% \\
\text { CI: } 0.503-0.806, p=0.037 \text { ) than did those in the lowest quartile. }\end{array}$ & $\begin{array}{l}\text { Higher consumption of vegetables and fruits is significantly associated with a lower } \\
\text { risk of depressive symptoms. }\end{array}$ & 5 \\
\hline [42] & $\begin{array}{l}\begin{array}{l}\text { Frequent consumption of fruits and vegetables is associated with improved health outcomes, } \\
\text { including mental health. }\end{array}\end{array}$ & $\begin{array}{c}\text { Frequent consumption of fruits and vegetables contributes to slower disablement } \\
\text { processes and might be an easily implementable way to improve the overall health of } \\
\text { older adults. }\end{array}$ & 6 \\
\hline [43] & $\begin{array}{l}\text { Fruit and vegetable consumption predicted an increased cognitive performance in older adults } \\
\text { including improved verbal recall, improved delayed verbal recall, improved digit span test } \\
\text { performance and improved verbal fluency; the effect of fruit consumption was much stronger } \\
\text { than the effect of vegetable consumption. Regarding mental health, fruit consumption was } \\
\text { significantly associated with better subjective quality of life and less depressive symptoms; } \\
\text { vegetable consumption, however, did not significantly relate to mental health. }\end{array}$ & $\begin{array}{l}\text { Consumption of fruits is associated with both improved cognitive and mental health in } \\
\text { older adults from non-Western developing countries, and consumption of vegetables is } \\
\text { associated with improved cognitive health only. Increasing fruit and vegetable } \\
\text { consumption might be one easy and cost-effective way to improve the overall health } \\
\text { and quality of life of older adults in non-Western developing countries. }\end{array}$ & 5 \\
\hline [44] & $\begin{array}{l}\text { Consumption of vegetables and fruits in the last } 3 \text { days was less likely to be associated with } \\
\text { depression (OR 0.11; } 95 \% \text { CI: } 0.02-0.45 \text { ) and subsyndromal depression (OR 0.10; CI 95\%: } \\
0.03-0.39 \text { ). }\end{array}$ & $\begin{array}{l}\text { Findings support the importance for a healthy consumption of vegetables and fruits for } \\
\text { the older adult population in Singapore. }\end{array}$ & 5 \\
\hline [45] & $\begin{array}{l}\text { Fixed effects regressions show that mental well-being (GHQ-12) responds in a dose-response } \\
\text { fashion to increases in both the quantity and the frequency of fruit and vegetables consumed. } \\
\text { This relationship is robust to the use of subjective well-being (life satisfaction) instead of mental } \\
\text { well-being. Increasing one's consumption of fruit and vegetables by one portion (on a day where } \\
\text { at least one portion is consumed) leads to a } 0.133 \text {-unit increase in mental well-being }(p<0.01) \text {. }\end{array}$ & $\begin{array}{l}\text { Persuading people to consume more fruits and vegetables may not only benefit their } \\
\text { physical health in the long-run, but also their mental well-being in the short-run. }\end{array}$ & 6 \\
\hline [35] & $\begin{array}{l}\text { Results of generalized estimating equations predicting mental-health-related quality of life } \\
\text { indicated that more frequent fruit consumption }(p=0.485) \text { was not, but more frequent vegetable } \\
\text { consumption ( } p=0.027) \text { was in the fully adjusted model associated with greater mental } \\
\text { health-related quality of life. Fruit and vegetable consumption }(p=0.033) \text { was associated with } \\
\text { greater mental-health-related quality of life only in the unadjusted model. More frequent fruit }(p \\
=0.566 \text { and } p=0.751 \text {, respectively), vegetable }(p=0.173 \text { and } p=0.399) \text {, and fruit and vegetable } \\
\text { consumption }(p=0.252 \text { and } p=0.634 \text {, respectively) did not significantly reduce the risk of major } \\
\text { depression and generalized anxiety disorder. }\end{array}$ & $\begin{array}{c}\text { The study did not find evidence that more frequent fruit and vegetable consumption } \\
\text { was associated with mental-health-related quality of life, depression, and anxiety. } \\
\text { However, more frequent vegetable consumption was associated with greater } \\
\text { mental-health-related quality of life. }\end{array}$ & 5 \\
\hline [46] & $\begin{array}{l}\text { Inverse association was found between mental distress and higher intake of fruits }(\beta=-0.64 \text {, } \\
95 \% \text { CI: }-0.89 \text { to }-0.39 ; p<0.001) \text {, vegetables }(\beta=-0.39,95 \% \text { CI: }-0.65 \text { to }-0.13 ; p<0.003) \text {. }\end{array}$ & $\begin{array}{l}\text { Study suggests beneficial association of Mediterranean diet and its elements (including } \\
\text { fruit and vegetables intake) and overall mental health, offering important implications } \\
\text { for public health provisions. }\end{array}$ & 5 \\
\hline
\end{tabular}


Table 3. Cont

\begin{tabular}{|c|c|c|c|}
\hline \multirow{2}{*}{ Ref. } & \multicolumn{2}{|l|}{ Findings } & \multirow{2}{*}{ Quality * } \\
\hline & Observation & Conclusion & \\
\hline [47] & $\begin{array}{l}\text { After adjusting for potential confounders such as age, body-mass-index (BMI), parity, } \\
\text { educational status, occupation, marital status, household hunger scale and household asset } \\
\text { index, there was an increasing trend across terciles of vegetable intake in the past month for the } \\
\text { HR-QoL }(p=0.0003) \text {, mental health (MH) }(p=0.001) \text { domain of the SF-36 and role emotional }(p< \\
\text { 0.0001) domain of the SF-36. The multivariate model results a significant increasing trend in the } \\
\text { adjusted mean scores of the HR-QoL }(p=0.04) \text {, MH }(p=0.001) \text { as well as } 4 \text { subscales of the SF-36 } \\
\text { [role-physical }(p=0.02) \text {, role-emotional }(p=0.05) \text {, emotional well-being }(p=0.002) \text { and vitality ( } p \\
<0.0001) \text { ] across terciles of the vegetable variety score. }\end{array}$ & $\begin{array}{l}\text { Results suggest a potential beneficial role of high vegetable intake and consumption of } \\
\text { more varied vegetables on HR-QoL. }\end{array}$ & 3 \\
\hline [48] & $\begin{array}{l}\text { After adjustment for confounding variables, the participants in the lower quartiles of total fruit } \\
\text { and vegetables, total vegetables, total fruits, citrus, other fruits and green leafy vegetables intake } \\
\text { were more likely to experience depression compared to those in the higher quartiles }(p<0.03) \text {. }\end{array}$ & $\begin{array}{l}\text { Lower intake of total fruit and vegetables and some of its specific subgroups might be } \\
\text { associated with depression. The findings support encouragement of fruit and } \\
\text { vegetables consumption as part of a healthy diet and highlight the importance of fruit } \\
\text { and vegetables consumption and a number of their subgroups in mitigating the chance } \\
\text { of depression. }\end{array}$ & 7 \\
\hline [49] & $\begin{array}{c}\text { Mixed linear models showed that higher baseline levels of psychological well-being were } \\
\text { associated with more fruit and vegetable consumption at baseline ( } \beta=0.05,95 \% \text { CI: } 0.02-0.08) \\
\text { and that fruit and vegetable consumption declined across time ( } \beta=0.01,95 \% \text { CI: } 0.02-0.004) \text {. } \\
\text { Psychological well-being interacted significantly with time such that individuals with higher } \\
\text { baseline psychological well-being had slower declines in fruit and vegetable consumption ( } \beta= \\
0.01,95 \% \text { CI: } 0.01-0.02 \text { ). Among individuals who initially met recommendations to consume } 5 \text { or } \\
\text { more servings of fruits and vegetables, higher baseline psychological well-being was associated } \\
\text { with } 11 \% \text { reduced risk of falling below recommended levels during follow-up (hazard ratio = } \\
0.89 ; 95 \% \text { CI: } 0.83-0.95) \text {. }\end{array}$ & $\begin{array}{l}\text { Psychological well-being may be a precursor to healthy behaviors such as eating a diet } \\
\text { rich in fruits and vegetables. Analyses also considered the likelihood of reverse } \\
\text { causality. }\end{array}$ & 6 \\
\hline [50] & $\begin{array}{c}\text { Controlling for covariates, raw fruit and vegetable intake (FVI) predicted reduced depressive } \\
\text { symptoms and higher positive mood, life satisfaction, and flourishing; processed FVI only } \\
\text { predicted higher positive mood. The top } 10 \text { raw foods related to better mental health were } \\
\text { carrots, bananas, apples, dark leafy greens like spinach, grapefruit, lettuce, citrus fruits, fresh } \\
\text { berries, cucumber, and kiwifruit. }\end{array}$ & $\begin{array}{l}\text { Raw FVI, but not processed FVI, significantly predicted higher mental health outcomes } \\
\text { when controlling for the covariates. Applications include recommending the } \\
\text { consumption of raw fruits and vegetables to maximize mental health benefits. }\end{array}$ & 2 \\
\hline [51] & $\begin{array}{l}\text { Individuals who were depressed at both times points had the highest proportion who failed to } \\
\text { consume any fruit }(31 \%) \text { or vegetables }(42 \%) \text { on the previous day. Fruit and vegetable } \\
\text { consumption did not predict of adult depression in fully adjusted models. }\end{array}$ & $\begin{array}{l}\text { Cross sectional associations existed for diet and adolescent depression only. For adult } \\
\text { depression association was subsequently attenuated on adjustment for other relevant } \\
\text { factors. }\end{array}$ & 5 \\
\hline [52] & Happiness was linearly associated with total fruit and vegetable intakes $(p=0.002)$. & $\begin{array}{l}\text { Maintaining good nutrition and increasing fruit and vegetable consumption may be } \\
\text { important for psychological health of older people. }\end{array}$ & 4 \\
\hline [53] & $\begin{array}{c}\text { Subjects who reported to consume a greater amount of fruit were associated with a lower risk of } \\
\text { depression (OR } 0.46 ; 95 \% \text { CI: } 0.26-0.84, p=0.011 \text { ) after adjustment for many possible } \\
\text { confounders. Similar results were obtained for women, while no statistically significant } \\
\text { differences emerged for men. }\end{array}$ & $\begin{array}{l}\text { Diet rich in olive oil and fruit, characteristics of Mediterranean diet, may protect against } \\
\text { the development of depressive symptoms in older age. }\end{array}$ & 4 \\
\hline
\end{tabular}


Table 3. Cont

\begin{tabular}{|c|c|c|c|}
\hline \multirow{2}{*}{ Ref. } & \multicolumn{2}{|l|}{ Findings } & \multirow{2}{*}{ Quality* } \\
\hline & Observation & Conclusion & \\
\hline [54] & $\begin{array}{l}\text { Women in the top quintile of fruit intake, compared with those in the bottom quintile, had } 57 \% \text {, } \\
50 \% \text {, and } 60 \% \text { lower odds of depression, anxiety, and psychological distress. Consumption of } \\
\text { vegetables was significantly associated with lower odds of depression (OR 0.65; } 95 \% \text { CI: } \\
0.46-0.93 \text { ) in women and lower odds of anxiety (OR 0.43; } 95 \% \text { CI: } 0.22-0.87 \text { ) in men. After } \\
\text { adjustment for potential confounders, women in the highest quintile of fruit and vegetables } \\
\text { intake, compared with those in the bottom quintile, had significantly lower odds of depression } \\
\text { (OR 0.55; } 95 \% \text { CI: } 0.37-0.80 \text { ) and psychological distress (OR 0.60; } 95 \% \text { CI: } 0.40-0.90) \text {. High intake } \\
\text { of total fruit and vegetables was associated with lower odds of psychological distress (OR 0.42; } \\
\text { 95\% CI: } 0.21-0.81 \text { ) in men. }\end{array}$ & $\begin{array}{l}\text { There was a significant inverse associations between high intake of fruit with } \\
\text { depression, anxiety, and psychological distress in Iranian women. High consumption of } \\
\text { vegetables was also associated with lower risk of depression and anxiety, in women and } \\
\text { men. In addition, high intake of total fruit and vegetable was associated with lower } \\
\text { odds of depression and psychological distress in women and men. }\end{array}$ & 6 \\
\hline [55] & $\begin{array}{l}\text { Vegetable intake were associated with increased sleep quality, which in turn was associated with } \\
\text { increased overall quality of life }(p<0.05) .\end{array}$ & $\begin{array}{l}\text { Results suggest possible relationships among the multiple health behaviors and their } \\
\text { associations with overall well-being. }\end{array}$ & 3 \\
\hline [56] & $\begin{array}{l}\text { Perceived ambiguity and cancer fatalism were negatively associated with fruit and vegetables } \\
\text { consumption }(p<0.001) \text { whereas health-related self-efficacy was positively associated with fruit } \\
\text { and vegetables consumption }(b=0.34, p<0.001) \text {. }\end{array}$ & $\begin{array}{l}\text { Individual choice influences fruit and vegetables consumption, but external control } \\
\text { beliefs, as measured by perceived ambiguity of cancer prevention recommendations } \\
\text { and cancer fatalism, and internal control beliefs, as measured by health-related } \\
\text { self-efficacy, may be important inputs to these decisions. }\end{array}$ & 7 \\
\hline [57] & $\begin{array}{l}\text { In India, those who consumed less than five servings of vegetables were respectively } 41 \% \text { (AOR } \\
=1.41 ; 95 \% \text { CI: } 0.60-3.33 \text { ) and } 57 \% \text { (AOR }=1.57 ; 95 \% \text { CI: } 0.93-2.64 \text { ) more likely to report } \\
\text { severe-extreme and mild-moderate depression during past } 30 \text { days compared to those who } \\
\text { consumed five servings a day. Regarding fruit consumption, compared to those who consumed } \\
\text { five servings a day, the odds of severe-extreme and mild-moderate self-reported depression were } \\
\text { respectively } 3.5 \text { times (AOR }=3.48 ; 95 \% \text { CI: } 1.216-10.01 \text { ) and } 45 \% \text { (AOR }=1.44 ; 95 \% \text { CI: } 0.89-2.32 \text { ) } \\
\text { higher in Bangladesh, and } 2.9 \text { times (AOR }=2.92 ; 95 \% \text { CI: } 1.12-7.64 \text { ) and } 42 \% \text { higher (AOR }=1.41 \text {; } \\
\text { 95\% CI: } 0.89-2.24 \text { ) in Nepal compared to those who consumed less than five servings a day } \\
\text { during last } 30 \text { days. }\end{array}$ & $\begin{array}{l}\text { Daily intake of less than five servings of fruit and vegetables was associated with higher } \\
\text { odds of depression. Nutrition programs aimed at promoting fruit and vegetables } \\
\text { consumption might prove beneficial to reduce the prevalence of depression in south } \\
\text { Asian population. }\end{array}$ & 5 \\
\hline [58] & $\begin{array}{l}\text { Baseline fruit and vegetable consumption considered separately or combined, was associated } \\
\text { with a lower prevalence of psychological distress even after adjustment for sociodemographic } \\
\text { characteristics and lifestyle risk factors. Baseline fruit and vegetable consumption, measured } \\
\text { separately or combined, was associated with a lower incidence of psychological distress in } \\
\text { minimally adjusted models. Most of these associations remained significant at medium levels of } \\
\text { intake but were no longer significant at the highest intake levels in fully adjusted models. }\end{array}$ & $\begin{array}{l}\text { Increasing fruit and vegetable consumption may help reduce psychological distress in } \\
\text { middle-aged and older adults. }\end{array}$ & 8 \\
\hline [59] & $\begin{array}{l}\text { Results indicate that the amount of fruit and vegetable consumption was positively associated } \\
\text { with happiness and inversely associated with depression. Happiness increased with any increase } \\
\text { to fruit and vegetable consumption, the strongest increase in the adjusted analysis was with } 6 \\
\text { servings of fruit and vegetables, with a coefficient of } 0.41 \text {. Depressive symptoms decreased with } \\
\text { any increase to fruit and vegetable consumption, the strongest decrease in the adjusted analysis } \\
\text { was with } 6 \text { servings of fruit and vegetables, with a coefficient of }-1.04 \text {. }\end{array}$ & $\begin{array}{l}\text { Healthier behavior patterns of fruit and vegetable consumption was associated with } \\
\text { higher happiness and lower depression scores among university students across } 28 \\
\text { countries. }\end{array}$ & 5 \\
\hline [60] & $\begin{array}{l}\text { The intake of green vegetables was associated with lower odds of having clinically-relevant } \\
\text { levels of depressive symptoms (OR 0.192, } p=0.016) \text {. }\end{array}$ & $\begin{array}{l}\text { Green vegetables, total FVI showed protective effects regarding clinically-relevant } \\
\text { levels of depressive symptoms. }\end{array}$ & 5 \\
\hline
\end{tabular}


Table 3. Cont

\begin{tabular}{|c|c|c|c|}
\hline \multirow{2}{*}{ Ref. } & \multicolumn{2}{|l|}{ Findings } & \multirow{2}{*}{ Quality ${ }^{*}$} \\
\hline & Observation & Conclusion & \\
\hline [61] & $\begin{array}{c}\text { For depression subtypes, statistically significantly positive associations of vegetable consumption } \\
\text { and adherence to the 5-a-day recommendation with current unspecified and current melancholic } \\
\text { major depressive disorder were found (OR 2.09; } 95 \% \text { CI: } 1.08-4.06 \text { and OR } 2.51 ; 95 \% \text { CI: } 1.21-5.21 \text {, } \\
\text { respectively; multivariable adjusted for demographic and other dietary factors) }\end{array}$ & $\begin{array}{l}\text { There is no consistent association between adherence to dietary recommendations and } \\
\text { major depressive disorder or subtypes of depression. }\end{array}$ & 5 \\
\hline [62] & $\begin{array}{l}\text { Mean positive affect increased linearly as a function of number of daily servings of fruits and } \\
\text { vegetables; the pattern of this relationship did not differ significantly for males and females. This } \\
\text { association remained statistically significant after controlling for demographic variables (age, sex, } \\
\text { and parent education levels); other diet variables (consumption of sugar containing beverages, } \\
\text { coffee or tea, and fat); and other health behaviors (exercise, sleep quality and smoking). Life } \\
\text { satisfaction and negative affect were not significantly related to fruit and vegetable consumption. }\end{array}$ & There is an association of fruits and vegetable consumption with well-being. & 3 \\
\hline [63] & $\begin{array}{l}\text { Participants in the lowest tertile of fruits and/or vegetables consumption had greater prevalence } \\
\text { of depressive symptoms ( } \mathrm{PR}=1.88 ; 95 \% \text { CI: } 1.39-2.55) \text { than those in the highest tertile. This } \\
\text { association was stronger with fruits (PR }=1.92 ; 95 \% \text { CI: } 1.46-2.53) \text { than vegetables ( } \mathrm{PR}=1.42 \\
95 \% \mathrm{CI}: 1.05-1.93 \text { ) alone. }\end{array}$ & $\begin{array}{l}\text { An inverse relationship between consumption of fruits and/or vegetables and } \\
\text { depressive symptoms was concluded. There is a need to implement strategies to } \\
\text { promote better diet patterns with potential impact on mental health. }\end{array}$ & 6 \\
\hline [64] & $\begin{array}{c}\text { High fruit or high vegetable consumption alone ( }>5 \text { times/week) was not significantly associated } \\
\text { with new depressive symptoms. Combining high fruit (OR 0.61; } 95 \% \text { CI: } 0.41-0.89 \text { ), vegetable } \\
\text { (OR 0.49; } 95 \% \text { CI: } 0.26-0.93 \text { ) or fruit and vegetable (OR } 0.39 ; 95 \% \text { CI: } 0.20-0.77 \text { ) consumption with } \\
\text { high leisure-time physical activity (LTPA) reduced the likelihood of developing subsequent new } \\
\text { depressive symptoms beyond LPTA alone. }\end{array}$ & $\begin{array}{l}\text { The simultaneous presence of several good lifestyle habits (high fruit and vegetable } \\
\text { consumption with high leisure-time physical activity) increases the beneficial effect of } \\
\text { reducing the risk of developing depressive symptoms in older adults. Thus, older } \\
\text { adults are encouraged to have as many good lifestyle habits as possible to reduce the } \\
\text { risk of depressive symptoms. }\end{array}$ & 7 \\
\hline [65] & $\begin{array}{c}\text { Measure of happiness was positively associated with the amount of fruit and vegetable } \\
\text { consumption }(p=0.02 \text {, and } 0.045 \text { respectively). Students who ate breakfast every day, more than } \\
8 \text { servings of fruit and vegetables daily, and had } 3 \text { meals in addition to } 1-2 \text { snacks per day had } \\
\text { the highest happiness score. }\end{array}$ & $\begin{array}{l}\text { Healthier behavior pattern (including fruit and vegetable consumption) was associated } \\
\text { with higher happiness scores among medical students. }\end{array}$ & 3 \\
\hline [66] & $\begin{array}{l}\text { Increased fruit and vegetable consumption was predictive of increased happiness, life } \\
\text { satisfaction, and well-being. They were up to } 0.24 \text { life-satisfaction points (for an increase of } 8 \\
\text { portions a day), which is equal in size to the psychological gain of moving from unemployment } \\
\text { to employment. Improvements occurred within } 24 \text { months. }\end{array}$ & $\begin{array}{l}\text { Eating certain foods is a form of investment in future happiness and well-being. The } \\
\text { implications of fruit and vegetable consumption are estimated to be substantial and to } \\
\text { operate within the space of } 2 \text { years. }\end{array}$ & 5 \\
\hline [67] & $\begin{array}{l}\text { In males, anxiety was correlated with lower daily intakes of fruits and vegetables }(\mathrm{r}=-0.216, p= \\
0.013) .\end{array}$ & A strict plant-based diet does not appear to negatively impact mood. & 2 \\
\hline [68] & $\begin{array}{l}\text { Fruit and vegetables consumption predicted greater eudaemonic well-being, curiosity, and } \\
\text { creativity at the between- and within-person levels. Young adults who ate more fruit and } \\
\text { vegetables reported higher average eudaemonic well-being, more intense feelings of curiosity, } \\
\text { and greater creativity compared with young adults who ate less fruit and vegetables. On days } \\
\text { when young adults ate more fruit and vegetables, they reported greater eudaemonic well-being, } \\
\text { curiosity, and creativity compared with days when they ate less fruit and vegetables. Fruit and } \\
\text { vegetables consumption also predicted higher positive effects, which mostly did not account for } \\
\text { the associations between fruit and vegetables and the other well-being variables. Lagged data } \\
\text { analyses showed no carry-over effects of fruit and vegetables consumption onto next-day } \\
\text { well-being (or vice versa). }\end{array}$ & $\begin{array}{l}\text { Fruit and vegetables consumption may be related to a broader range of well-being } \\
\text { states than signal human flourishing in early adulthood. }\end{array}$ & 4 \\
\hline
\end{tabular}


Table 3. Cont

\begin{tabular}{|c|c|c|c|}
\hline \multirow{2}{*}{ Ref. } & \multicolumn{2}{|l|}{ Findings } & \multirow{2}{*}{ Quality * } \\
\hline & Observation & Conclusion & \\
\hline [69] & $\begin{array}{l}\text { Fruit and vegetable consumption at each cycle was inversely associated with next-cycle } \\
\text { depression }(\beta=-0.03,95 \% \text { CI: }-0.05 \text { to }-0.01, p<0.01) \text { and psychological distress }(\beta=-0.03 \text {, } \\
95 \% \text { CI: }-0.05 \text { to }-0.02, p<0.0001) \text {. However, once models were adjusted for other health-related } \\
\text { factors, these associations were attenuated ( } \beta=-0.01,95 \% \text { CI: }-0.04 \text { to } 0.02, p=0.55 ; \beta=-0.00 \text {, } \\
95 \% \text { CI: }-0.03 \text { to } 0.02, p=0.78 \text { for models predicting depression and distress, respectively). }\end{array}$ & $\begin{array}{c}\text { Findings suggest that relations between fruit and vegetable intake, other health-related } \\
\text { behaviors and depression are complex. }\end{array}$ & 8 \\
\hline [70] & $\begin{array}{l}\text { Significant associations were found between vegetables intake (green salad) and physical health } \\
\text { days for Hispanics and fruit and vegetables intake (green salad, fruit, and fruit juice) and } \\
\text { self-reported health for Chinese. }\end{array}$ & $\begin{array}{l}\text { There is a need to promote healthy living behaviors among aging NYC racial/ethnic } \\
\text { populations }\end{array}$ & 5 \\
\hline [71] & $\begin{array}{l}\text { Men who experienced mild to moderate levels of stress were less likely to consume vegetables } \\
\text { and fruit }(p<0.05 \text { ) compared with their unstressed counterparts. The trend analysis results } \\
\text { indicated significant dose-response patterns in the relationship between stress level and } \\
\text { consumption of vegetables and fruit (negative trend) (adjusted OR } 0.50 \text {; } 95 \% \text { CI: } 0.48-0.87 ; p< \\
0.05 \text { ). For female students significant dose-response trend was found in the relationship between } \\
\text { stress levels and the consumption of vegetables and fruit (both negative trends) }(p<0.01 \text { ). }\end{array}$ & $\begin{array}{l}\text { There is a difference in food selection patterns between stressed male and female } \\
\text { students, with stress being a more significant predictor of unhealthy food selection } \\
\text { among male students. }\end{array}$ & 4 \\
\hline [72] & $\begin{array}{l}\text { Consumers fulfilling the } 5 \text {-a-day recommendation had lower odds of being highly or moderately } \\
\text { distressed than individuals consuming less fruit and vegetables (moderate vs. low distress: OR } \\
0.82 ; 95 \% \text { CI: } 0.69-0.97 \text {; high vs. low distress: OR } 0.55 ; 95 \% \text { CI: } 0.41-0.75 \text { ). }\end{array}$ & $\begin{array}{c}\text { Daily intake of } 5 \text { servings of fruit and vegetable was associated with lower } \\
\text { psychological distress. }\end{array}$ & 5 \\
\hline [73] & $\begin{array}{c}\text { For females fresh fruits }(-0.085 ; p<0.001) \text {, salad/raw vegetables }(-0.048 ; p<0.001) \text {, cooked } \\
\text { vegetables }(-0.061 ; p<0.001) \text { intake were respectively negatively associated with Perceived } \\
\text { Stress Score. For both sexes, consuming fresh fruits }(-0.111 ; p<0.001 \text { for females; }-0.074 ; p= \\
0.047 \text { for males), salads }(-0.071 ; p<0.001 \text { for females; }-0.091 ; p=0.014 \text { for males), cooked } \\
\text { vegetables }(-0.072 ; p<0.001 \text { for females; }-0.089 ; p=0.017 \text { for males) was significantly negatively } \\
\text { associated with perceived stress and depressive symptoms scores. }\end{array}$ & $\begin{array}{l}\text { The associations between consuming 'healthy' foods and lower depressive symptoms } \\
\text { and perceived stress among male and female students in three UK countries suggest } \\
\text { that interventions to reduce depressive symptoms and stress among students could also } \\
\text { result in the consumption of healthier foods and/or vice versa. }\end{array}$ & 5 \\
\hline [74] & $\begin{array}{c}\text { Analysis showed reduced odds of depressive symptoms OR } 0.86 \text { ( } 95 \% \text { CI: } 0.79-0.95, p=0.001) \\
\text { among women who ate } \geq 2 \text { of fruit/day and OR } 0.79 \text { ( } 95 \% \text { CI: } 0.67-0.93, p=0.007) \text { among women } \\
\text { who ate } \geq 5 \text { vegetables/day, even after adjustment for several factors including smoking, alcohol, } \\
\text { body mass index, physical activity, marital status, education, energy, fish intake, and } \\
\text { comorbidities. }\end{array}$ & $\begin{array}{l}\text { Increasing fruit consumption may be one important factor for reducing both the } \\
\text { prevalence and incidence of depressive symptoms in mid-age women. }\end{array}$ & 6 \\
\hline [75] & $\begin{array}{l}\text { Higher Beck Depression Inventory scores correlated with lower fruit and vegetable consumption } \\
(\mathrm{r}=-0.20, p=0.006) \text {. Women reporting a history of treatment for depression showed lower } \\
\text { levels of fruit and vegetable consumption (mean daily servings }=2.0[1.3] \text { vs. } 2.5[1.3] \\
\text { respectively, } p=0.03) \text {. Participants reporting current antidepressant use (versus non-users) did } \\
\text { not differ on dietary habits. }\end{array}$ & $\begin{array}{l}\text { Fruit and vegetable consumption partially mediated associations between depression } \\
\text { and time to cardiovascular disease events. }\end{array}$ & 6 \\
\hline [37] & Depressive symptoms were not associated with fruit and vegetables intake $(p>0.05)$. & $\begin{array}{l}\text { Future studies should explore the mechanisms linking the identified associations } \\
\text { between depressive symptoms and dietary intake, such as the role of emotional eating. }\end{array}$ & 6 \\
\hline [76] & $\begin{array}{l}\text { After adjustment for potential confounders, the AHEI score was inversely associated with } \\
\text { recurrent depressive symptoms in a dose-response fashion in women ( } p=0.001 \text {; for } 1 \mathrm{SD} \text { in } \\
\text { AHEI score; OR 0.59; } 95 \% \text { CI: } 0.47-0.75) \text { but not in men, while among its components vegetable } \\
\text { and fruit intake were significant. }\end{array}$ & Poor diet may be a risk factor for future depression in women. & 6 \\
\hline
\end{tabular}


Table 3. Cont

\begin{tabular}{|c|c|c|c|}
\hline \multirow{2}{*}{ Ref. } & \multicolumn{2}{|l|}{ Findings } & \multirow{2}{*}{ Quality * } \\
\hline & Observation & Conclusion & \\
\hline [38] & $\begin{array}{l}\text { No significant associations were found between prevalence of distress and vegetable and/or fruit } \\
\text { intake ( } p=0.911 \text { for males; } p=0.908 \text { for females). }\end{array}$ & Psychological distress is not associated with reduced intake fruit and vegetables. & 5 \\
\hline [77] & $\begin{array}{l}\text { Greater fruit and vegetable intake was significantly associated with lower odds of depression } \\
\text { (OR 0.72; } 95 \% \text { CI: } 0.71-0.75 \text { ) - for all } 5 \text { waves. Perceived poor mental health status and previous } \\
\text { diagnosis of a mood disorder and anxiety disorder also demonstrated statistically significant } \\
\text { inverse associations with fruit and vegetable intake (all } p<0.05) \text {. } \\
\text { In the first wave, greater fruit and vegetable intake was significantly associated with lower odds } \\
\text { of depression (OR 0.85; } 95 \% \text { CI: } 0.78-0.92 \text { ). A combined estimate of all } 5 \text { waves demonstrated } \\
\text { similar results (OR } 0.72 ; 95 \% \text { CI: } 0.71-0.75) \text {. Relative to those with the lowest fruit and vegetable } \\
\text { intake, those with the greatest fruit and vegetable intake also had significantly lower odds of } \\
\text { suffering from distress (OR } 0.87 ; 95 \% \text { CI: } 0.78-0.98 \text { ). These results were consistent across other } \\
\text { waves. Perceived poor mental health status and previous diagnosis of a mood disorder and } \\
\text { anxiety disorder also demonstrated statistically significant inverse associations with fruit and } \\
\text { vegetable intake (all } p<0.05 \text { ). }\end{array}$ & $\begin{array}{l}\text { Findings suggest a potentially important role of a healthy diet in the prevention of } \\
\text { depression and anxiety. }\end{array}$ & 5 \\
\hline [78] & $\begin{array}{l}\text { There was no significant difference }(p>0.05) \text { of intake of vegetable products and dishes in } \\
\text { women with depression compared with women without depression. The regression model } \\
\text { indicated among others that increased intakes per kilojoule of vegetables }(p=0.015) \text { are } \\
\text { associated with lower odds of having depression. }\end{array}$ & The results confirm a collective effect of diet on mood. & 6 \\
\hline [79] & $\begin{array}{l}\text { After adjustments for potentially confounding factors, the odds ratios of having mild and severe } \\
\text { depressive symptoms by increasing levels of tomatoes/tomato products were } 1.00,0.54 \text {, and } 0.48 \\
(p<0.01) \text {. No relationship was observed between intake of other kinds of vegetables and } \\
\text { depressive symptoms. }\end{array}$ & $\begin{array}{l}\text { Tomato-rich diet is independently related to lower prevalence of depressive symptoms } \\
\text { and may have a beneficial effect on the prevention of depressive symptoms. }\end{array}$ & 6 \\
\hline [80] & $\begin{array}{l}\text { Dietary intake of inter alia fruits and vegetables was significantly higher in the low-stress group } \\
\text { than in high-stress group. There was an inverse association between stress level and intake of } \\
\text { fruits and vegetables (OR } 0.83 ; 95 \% \text { CI: } 0.76-0.90)\end{array}$ & $\begin{array}{l}\text { The results showed a significant positive association between dietary intake and stress. } \\
\text { There must be a special attention to dietary intake in stress management program of } \\
\text { high-stress individuals, and in dietary recommendations, psychologic aspects should } \\
\text { be considered. }\end{array}$ & 5 \\
\hline [81] & $\begin{array}{l}\text { Analyses of same-day within-person associations revealed that on days when young adults } \\
\text { experienced greater positive affect, they reported eating more servings of fruit }(p=0.002) \text { and } \\
\text { vegetables }(p<0.001) \text {. Results of lagged analysis showed that fruits and vegetables predicted } \\
\text { improvements in positive affect the next day, suggesting that healthy foods were driving } \\
\text { affective experiences and not vice versa. Meaningful changes in positive affect were observed } \\
\text { with the daily consumption of approximately } 7-8 \text { servings of fruit or vegetables. }\end{array}$ & $\begin{array}{l}\begin{array}{c}\text { Eating fruit and vegetables may promote emotional well-being among healthy young } \\
\text { adults. }\end{array}\end{array}$ & 3 \\
\hline [82] & $\begin{array}{l}\text { In cross-sectional data, happiness and mental health rise in an approximately dose-response way } \\
\text { with the number of daily portions of fruit and vegetables. Well-being peaks at approximately } 7 \\
\text { portions per day. It was documented for seven measures of well-being (life satisfaction, } \\
\text { WEMWBS mental well-being, GHQ mental disorders, self-reported health, happiness, } \\
\text { nervousness, and feeling low). The pattern is robust to adjustment for a large number of other } \\
\text { demographic, social and economic variables. }\end{array}$ & $\begin{array}{l}\begin{array}{l}\text { There is a positive association between eating fruit and vegetables and having high } \\
\text { mental well-being. }\end{array}\end{array}$ & 4 \\
\hline
\end{tabular}


Table 3. Cont

\begin{tabular}{|c|c|c|c|}
\hline \multirow{2}{*}{ Ref. } & \multicolumn{2}{|l|}{ Findings } & \multirow{2}{*}{ Quality * } \\
\hline & Observation & Conclusion & \\
\hline [83] & $\begin{array}{l}\text { Compared to the regional nutrition survey data, a greater proportion of study participants (the } \\
\text { members of Mood Disorders Association of British Columbia) consumed fewer of the } \\
\text { recommended servings of vegetables and fruits }(p<0.05) \text {. }\end{array}$ & $\begin{array}{l}\text { The adults with mood disorders could benefit from nutritional interventions to improve } \\
\text { diet quality. }\end{array}$ & 4 \\
\hline [84] & $\begin{array}{l}\text { Fruit and vegetable consumption was lower in depressed individuals, than in comparison } \\
\text { individuals, that remained significant in multivariable models. }\end{array}$ & $\begin{array}{l}\text { These results may indicate the importance of components of fruits and vegetables, } \\
\text { including antioxidants, rather than dietary supplements. }\end{array}$ & 6 \\
\hline [85] & $\begin{array}{l}\text { In a regression model that controlled for demographic, socio-economic, lifestyle and } \\
\text { disease/health-related variables but not cognitive status, both fruits (OR } 0.66 ; 95 \% \text { CI: } 0.45-0.98, p \\
=0.038 \text { ) and vegetables (OR } 0.38 ; 95 \% \text { CI: } 0.17-0.86, p=0.021 \text { ) were protective against depressive } \\
\text { symptoms } 4 \text { years later. When the same regression model was also adjusted for cognitive status, } \\
\text { only vegetables (OR } 0.40,95 \% \text { CI: } 0.17-0.95, p=0.039 \text { ) were protective against depressive } \\
\text { symptoms. }\end{array}$ & $\begin{array}{l}\text { More frequent consumption of vegetables seems to be protective against depressive } \\
\text { symptoms in the elderly. }\end{array}$ & 7 \\
\hline [36] & Patients who ate fruit more frequently tended to have a better quality of life. & There is an association between food pattern and quality of life in this population. & 2 \\
\hline [39] & Neither mental nor physical health was associated with fruit and vegetable intake $(p<0.05)$. & $\begin{array}{l}\begin{array}{l}\text { Fruit and vegetable consumption may be too specific to represent an individual's } \\
\text { overall diet. }\end{array} \\
\end{array}$ & 4 \\
\hline [86] & Depressive symptoms were related to a lower consumption of vegetables/fruit. & $\begin{array}{l}\text { Depressive symptoms may affect unhealthy food choices. The relations between } \\
\text { negative emotions such as depressive symptoms and food consumption are most likely } \\
\text { bidirectional: food consumed affects mood and mood affects food choices. }\end{array}$ & 6 \\
\hline [87] & $\begin{array}{l}\text { The consumption of vegetables is more prevalent among people with low or moderate } \\
\text { depression than those with severe depression }(p<0.05) .\end{array}$ & $\begin{array}{l}\text { Unhealthy dietary choices seems to promote depression. Efforts to lower the prevalence } \\
\text { of depression in the elderly should target on the factors such as dietary habits. }\end{array}$ & 6 \\
\hline [88] & $\begin{array}{l}\text { In men, attempters }(\mathrm{n}=92) \text { had a high odds of low consumption of vegetables (OR } 2.47,95 \% \text { CI: } \\
\text { 1.19-5.15). In women, attempters }(\mathrm{n}=275) \text { had a high odds of insufficient fruit consumption (OR } \\
2.36,95 \% \text { CI: } 1.15-4.85) .\end{array}$ & $\begin{array}{l}\text { The data suggest that fruits and vegetables were significantly under-consumed in } \\
\text { adults who had ever attempted suicide. }\end{array}$ & 6 \\
\hline [89] & $\begin{array}{l}\text { For male students, none of the food consumption groups were associated with perceived stress } \\
\text { or depressive symptoms. In females, perceived stress and depressive symptoms were associated } \\
\text { with less frequent consumption of fruits/vegetables. }\end{array}$ & $\begin{array}{l}\text { Consistent associations were observed between unhealthy food consumption and } \\
\text { depressive symptoms and perceived stress among female students from three European } \\
\text { countries, but not among male students. Efforts to reduce depressive symptoms and } \\
\text { stress among female students may also lead to the consumption of healthier foods } \\
\text { and/or vice-versa. }\end{array}$ & 4 \\
\hline [90] & $\begin{array}{l}\text { Single mothers had lower intake of fruits and vegetables and lower self-worth compared to the } \\
\text { married and cohabiting mothers, controlling for age, education and BMI. }\end{array}$ & $\begin{array}{l}\text { A lower sense of self-worth and lower intake of fruit and vegetables in single mothers } \\
\text { could be seen in the context of the social disadvantages and less social support. }\end{array}$ & 6 \\
\hline [91] & $\begin{array}{l}\text { A high level of dispositional optimism was associated with higher intakes of fruit }(p=0.01) \text {, and } \\
\text { vegetables }(p=0.01) \text {, independently from age, education, living arrangement, self-rated health, } \\
\text { cardiovascular disease, diabetes mellitus, cancer, and body mass index, as well as total energy } \\
\text { intake. }\end{array}$ & $\begin{array}{l}\text { Dispositional optimism in elderly men is associated with healthy lifestyle and dietary } \\
\text { habits. }\end{array}$ & 7 \\
\hline [92] & $\begin{array}{l}\text { Stepwise logistic regression models found that frequency of consumption of fresh fruit had } \\
\text { apparently independent effects on perceived stress, whereas the intake level of fresh fruit was } \\
\text { significantly associated with depression. }\end{array}$ & $\begin{array}{l}\text { The link between food consumption frequency, perceived stress and depression } \\
\text { suggests that diet intervention may be considered a mediate strategy integrated in } \\
\text { psychology prevention program among normal population of the college. }\end{array}$ & 5 \\
\hline
\end{tabular}


Table 3. Cont.

\begin{tabular}{|c|c|c|c|}
\hline \multirow{2}{*}{ Ref. } & \multicolumn{2}{|l|}{ Findings } & \multirow{2}{*}{ Quality } \\
\hline & Observation & Conclusion & \\
\hline [93] & $\begin{array}{l}\text { Both women and men above the upper quartile for optimism more often ate fresh vegetables and } \\
\text { salads (women } 6 \% / \text { men } 57 \%) \text {, berries }(23 \% / 9 \%) \text {, fruit }(67 \% / 42 \%) \text {, than those below the lower } \\
\text { quartile }(56 \% / 31 \%, 14 \% / 5 \% \text {, and } 52 \% / 26 \% \text {, respectively) with women in higher proportion than } \\
\text { in men in each case. }\end{array}$ & Lack of optimism is associated with a cluster of unhealthy dietary and other habits. & 7 \\
\hline [94] & $\begin{array}{l}\text { Women having poor mental health were less likely than their healthier counterparts to report } \\
\text { consuming fresh vegetables, fresh fruits on a daily basis. Men having poor mental health } \\
\text { reported consuming less frequently fresh fruits. These results remained statistically significant in } \\
\text { the fully adjusted model. }\end{array}$ & The results suggest that poor mental health is associated with unhealthy food habits. & 5 \\
\hline [95] & $\begin{array}{l}\text { The mood of females was significantly better when more fruit/vegetables were consumed } \\
(\mathrm{F}(2,157)=12.39, p<0.001) \text {. There was no association between the amount consumed by males } \\
\text { and scores on the GHQ }[\mathrm{F}(2,163)=1.09 \text {, NS]. In females a lower intake of fruit/vegetables was } \\
\text { associated with higher levels of anxiety }(\mathrm{F}(2,157)=8.78, p<0.002) \text {, a relationship not found in } \\
\text { males }(\mathrm{F}(2,163)=0.29, \mathrm{NS}) \text {. Females who consumed greater amounts of fruit/vegetables were less } \\
\text { depressed }[\mathrm{F}(2,157)=12.77, p<0.0001] \text {, although there was no such relationship in males } \\
(\mathrm{F}(2,163)=1.23, \mathrm{NS}) .\end{array}$ & $\begin{array}{l}\text { Eating large amounts of fruit and vegetables were less likely to be anxious or depressed; } \\
\text { the relationship existed irrespective of age and social background. }\end{array}$ & 4 \\
\hline
\end{tabular}




\section{Discussion}

The systematic review of the observational studies analyzing the association between the intake of fruit and vegetable and mental health in adults revealed the possible beneficial influence of the indicated products. This association was studied for various aspects of mental health, ranging from general and mental well-being $[35,45,49,62,68,77,82,84,94]$, quality of life $[36,39,42,43,47,55,70]$, sleep quality [55], life satisfaction [50,66,82], flourishing [50], mood [50,62,68,77,81], self-efficacy [56], curiosity [68], creativity [68], optimism [91,93], self-esteem [90], stress [40,48,67,71,73,80,89,92], nervousness [82], or happiness [52,59,65,82], to anxiety [35,48,50,54,67], minor psychiatric disorders [46], distress $[38,46,54,58,69,72,77,82]$, depressive symptoms $[37,41,43,50,51,53,59,60,63,64,73,74,76,79,85-87$, 89], depression [35,42,44,48,54,57,61,67,69,75,77,78,83,84,92], or attempted suicide [88].

Moreover, the indicated effect was studied and stated not only for fresh fruits and vegetables [35-95] but also for fruit and vegetable products, such as juices $[37,39,40,48,49,51,52,54,56,60,61,65,70,72,82,83$, $88,92,94]$, dried $[37,49,54,66]$, and canned fruits and vegetables [37,38,49,50,58,65,66], salads [39,40,44, $49,52,54,58,61,62,72-74,89,93,94]$, soups [39,40,62], or ketchup [39,79], and even for potatoes in some studies that included it to the total intake of fruits and vegetables $[39,74]$. This corresponds to the general conclusions of some studies that not only the intake of fruit and vegetable should be increased, but also at least 5 portions of fruits or vegetables must be taken daily as recommended to observe a positive influence on the general mental health $[57,72]$.

The reason behind promoting the consumption of 5 portions of fruits or vegetables daily is that the Food and Agriculture Organization of the United Nations (FAO) and WHO have recommended to consume a minimum of $400 \mathrm{~g}$ of fruits and vegetables per day, excluding potatoes and other starchy tubers, with an estimated serving size of $80 \mathrm{~g}$ [96]. This recommendation is also supported by a number of prominent experts, boards, and associations, such as the National Health Service (NHS) in Great Britain [97], American Heart Association (AHA) [98], Centers for Disease Control and Prevention (CDC) [99], and Office of Disease Prevention and Health Promotion (ODPHP) in the United States of America [100]. Moreover, not only the role of raw fruits and vegetables but also that of the processed fruits and vegetables, including frozen, canned, or cooked ones [101], as well as juices [102], is emphasized to meet the recommended intake.

Increasing the intake of fruits and vegetables to the recommended level may result in a noticeable and measurable effect, as was stated in some included studies. An increase in the consumption of fruits and vegetables by one portion a day leads to a 0.133 -unit improvement in the mental well-being assessed by GHQ-12 scale [45], while the consumption of 7-8 servings a day leads to meaningful changes in positive affect [81] and consumption of 8 portions a day leads to a 0.24 -unit increase in life satisfaction (equivalent to the psychological gain of moving from unemployed status to employed) [66]. However in general, any increase in the consumption of fruits and vegetables results in the improvement of well-being, enhances happiness, and decreases depressive symptoms, with the strongest effect observed for 6 servings a day [59], 7 servings a day [82], or more than 8 servings a day (combined with breakfast every day and 3 meals in addition to 1-2 snacks per day) [65], depending on the studied group.

It must be indicated that among the included studies, some highlighted not only the general effect of fruits and vegetables but also the influence of specific types, such as citrus [48], berries [93], green leafy vegetables [48], green salad [70], and tomatoes [79]. Simultaneously, among the raw fruits and vegetables, the following were indicated as specially related to better mental health: Bananas, apples, citrus, berries, grapefruit, kiwifruit, carrots, lettuce, cucumber, and green leafy vegetables, particularly spinach [50]. Authors of the indicated studies have no definite explanation why those fruits or vegetables may be especially valuable, similarly as the general mechanism of the influence on mental health is still unknown [18]. However, it may be supposed that the positive influence should be attributed to a specific nutritional value, as a combination of high content of compounds positive for mental health and, at the same time, low content of those negative for mental health, as indicated by LaChance and Ramsey [12]. However, such assumption does not take into account the potential interactions between nutrients in food product and between food products in the diet, so it must 
be also emphasized that in fact estimating the independent effect of only one type of food products (fruits and vegetables), or nutrients may be hard to conduct, due to other food products, or nutrients, that are interfering.

While presenting the results of the included studies analyzing the association between the intake of fruit and vegetable and mental health in adults, it must be emphasized that the risk of bias varied from very high to low (defined based on the commonly assumed criteria [34]) as shown by the total NOS score. Taking this into account, the highest attention must be paid to those studies interpreted as having a low risk of bias and the highest quality. Within such studies, it was observed that high total intake of fruits and vegetables and some of their specific subgroups, including berries, might be associated with a high level of optimism [93], while this association is independent of interfering factors [91]. High intake of fruits and vegetables was also associated with a higher level of self-efficacy [56], as well as a low level of psychological distress [58], ambiguity, and cancer fatalism [56]. In addition, it was observed that high total intake of fruits and vegetables and some of their specific subgroups, including citrus and green leafy vegetables, might be associated with a lower risk of depression [48], but other health-related factors may also play a role in this association $[69,85]$. Furthermore, it was highlighted that increasing physical activity may be necessary to benefit from the positive effect of the consumption of fruit and vegetable to protect against depressive symptoms [64].

In spite of the fact that the vast majority of the included studies presented compatible conclusions that mental health benefits can be attained with increased consumption of fruits and vegetables, some limitations of this systematic review must be indicated. The main issue results from the fact, that association between fruit or vegetables intake and mental health does not allow to state unambiguously that the intake influences mental health, as the reverse causation is also possible, so only based on experimental study it may be concluded with no doubts, that such influence exists. Moreover, it must be emphasized that no uniform definition of fruit and vegetables was established in analyzed studies as various fruit and vegetable products were either included or excluded from this group, that may also have influenced the observed association. Last but not least, the included studies were conducted using various methodologies, in different populations, and involved diverse measures of intake of fruits and vegetables. Therefore, further studies should be conducted covering all the aspects of mental health in various populations and using the similar methodology, to analyze the association between the consumption of fruits and vegetables and mental health in detail.

\section{Conclusions}

The vast majority of the included studies indicated that the intake of fruits and/or vegetables and their specific subgroups, as well as processed fruits and vegetables, seems to have a positive influence on mental health. Therefore, the general recommendation to consume at least 5 portions of fruit and vegetable a day may be beneficial also for mental health.

Supplementary Materials: The following are available online at http://www.mdpi.com/2072-6643/12/1/115/s1, Table S1: Full electronic search strategy applied for PubMed and Web of Science databases, Table S2: List of studies included to the systematic review (in alphabetic order), Table S3: Characteristics of the study participants for the studies included to the systematic review, Table S4: Detailed results of the quality assessment based on the total score for the Newcastle-Ottawa Scale for categories of selection, comparability and exposure/outcome.

Author Contributions: D.G. (Dominika Głabska) and D.G. (Dominika Guzek) made study conception and design; D.G. (Dominika Głąbska), D.G. (Dominika Guzek), B.G. and K.G. performed the research; D.G. (Dominika Głąbska), D.G. (Dominika Guzek), B.G and K.G. analyzed the data; D.G. (Dominika Głąbska), D.G. (Dominika Guzek), B.G and K.G. wrote the paper. All authors have read and agreed to the published version of the manuscript.

Funding: The study was financed by the Certified Product 'CP' Quality System (CEN.DRR.WFP.705.350.2018.MR.3) within the National Polish Promotion Fund for Fruits and Vegetables Consumption.

Conflicts of Interest: The authors declare no conflict of interest. The funders had no role in the design of the study, in the collection, analyses, or interpretation of data, in the writing of the manuscript, or in the decision to publish the results. 


\section{References}

1. International Statistical Classification of Diseases and Related Health Problems 10th Revision, ICD-10 Version. 2016. Available online: https://icd.who.int/browse10/2016/en (accessed on 31 October 2019).

2. WHO. Mental Health Action Plan 2013-2020. Available online: https://icd.who.int/browse10/2016/en (accessed on 31 October 2019).

3. WHO. Mental Health Action Plan 2013-2020; World Health Organization: Geneva, Switzerland, 2013; pp. 1-48.

4. WHO. Mental Health Action Plan 2013-2020. Comprehensive Mental Health Action Plan $2013-2020$. Available online: Apps.who.int/gb/ebwha/pdf_files/WHA66/A66_R8-en.pdf?ua=1 (accessed on 31 October 2019).

5. Wattick, R.A.; Hagedorn, R.L.; Olfert, M.D. Relationship between Diet and Mental Health in a Young Adult Appalachian College Population. Nutrients 2018, 10, 957. [CrossRef] [PubMed]

6. Abbasalizad Farhangi, M.; Dehghan, P.; Jahangiry, L. Mental health problems in relation to eating behavior patterns, nutrient intakes and health related quality of life among Iranian female adolescents. PLoS ONE 2018, 13, e0195669. [CrossRef] [PubMed]

7. O’Neil, A.; Quirk, S.E.; Housden, S.; Brennan, S.L.; Williams, L.J.; Pasco, J.A.; Berk, M.; Jacka, F.N. Relationship between diet and mental health in children and adolescents: A systematic review. Am. J. Public Health 2014, 104, 31-42. [CrossRef] [PubMed]

8. Parletta, N.; Zarnowiecki, D.; Cho, J.; Wilson, A.; Bogomolova, S.; Villani, A.; Itsiopoulos, C.; Niyonsenga, T.; Blunden, S.; Meyer, B.; et al. A Mediterranean-style dietary intervention supplemented with fish oil improves diet quality and mental health in people with depression: A randomized controlled trial (HELFIMED). Nutr. Neurosci. 2019, 22, 474-487. [CrossRef] [PubMed]

9. Jacka, F.N.; O’Neil, A.; Opie, R.; Itsiopoulos, C.; Cotton, S.; Mohebbi, M.; Castle, D.; Dash, S.; Mihalopoulos, C.; Chatterton, M.L.; et al. A randomised controlled trial of dietary improvement for adults with major depression (the 'SMILES' trial). BMC Med. 2017, 15, 23. [CrossRef] [PubMed]

10. Tapsell, L.C.; Neale, E.P.; Satija, A.; Hu, F.B. Foods, Nutrients, and Dietary Patterns: Interconnections and Implications for Dietary Guidelines. Adv. Nutr. 2016, 7, 445-454. [CrossRef]

11. LaChance, L.; Ramsey, D. Food, mood, and brain health: Implications for the modern clinician. Mo. Med. 2015, 112, 111-115.

12. LaChance, L.R.; Ramsey, D. Antidepressant foods: An evidence-based nutrient profiling system for depression. World J. Psychiatry 2018, 8, 97-104. [CrossRef]

13. Liu, X.; Yan, Y.; Li, F.; Zhang, D. Fruit and vegetable consumption and the risk of depression: A meta-analysis. Nutrition 2016, 32, 296-302. [CrossRef]

14. Saneei, P.; Saghafian, F.; Esmaillzadeh, A.R. “Fruit and vegetable consumption and the risk of depression: A meta-analysis": Further analysis is required. Nutrition 2016, 32, 1162-1163. [CrossRef]

15. Kawada, T.R. Fruit and vegetable consumption and the risk of depression: A meta-analysis. Nutrition 2018, 45, 147. [CrossRef] [PubMed]

16. Saghafian, F.; Malmir, H.; Saneei, P.; Milajerdi, A.; Larijani, B.; Esmaillzadeh, A. Fruit and vegetable consumption and risk of depression: Accumulative evidence from an updated systematic review and meta-analysis of epidemiological studies. Br. J. Nutr. 2018, 119, 1087-1101. [CrossRef] [PubMed]

17. Tuck, N.J.; Farrow, C.; Thomas, J.M. Assessing the effects of vegetable consumption on the psychological health of healthy adults: A systematic review of prospective research. Am. J. Clin. Nutr. 2019, 110, 196-211. [CrossRef] [PubMed]

18. Rooney, C.; McKinley, M.C.; Woodside, J.V. The potential role of fruit and vegetables in aspects of psychological well-being: A review of the literature and future directions. Proc. Nutr. Soc. 2013, 72, 420-432. [CrossRef]

19. Aune, D.; Giovannucci, E.; Boffetta, P.; Fadnes, L.T.; Keum, N.; Norat, T.; Greenwood, D.C.; Riboli, E.; Vatten, L.J.; Tonstad, S. Fruit and vegetable intake and the risk of cardiovascular disease, total cancer and all-cause mortality-a systematic review and dose-response meta-analysis of prospective studies. Int. J. Epidemiol. 2017, 46, 1029-1056. [CrossRef]

20. Knüppel, A.; Shipley, M.J.; Llewellyn, C.H.; Brunner, E.J. Sugar intake from sweet food and beverages, common mental disorder and depression: Prospective findings from the Whitehall II study. Sci. Rep. 2017, 7, 6287. [CrossRef] 
21. Xu, H.; Li, S.; Song, X.; Li, Z.; Zhang, D. Exploration of the association between dietary fiber intake and depressive symptoms in adults. Nutrition 2018, 54, 48-53. [CrossRef]

22. Gangwisch, J.E.; Hale, L.; Garcia, L.; Malaspina, D.; Opler, M.G.; Payne, M.E.; Rossom, R.C.; Lane, D. High glycemic index diet as a risk factor for depression: Analyses from the Women's Health Initiative. Am. J. Clin. Nutr. 2015, 102, 454-463. [CrossRef]

23. Sahraian, A.; Ghanizadeh, A.; Kazemeini, F. Vitamin C as an adjuvant for treating major depressive disorder and suicidal behavior, a randomized placebo-controlled clinical trial. Trials 2015, 16, 94. [CrossRef]

24. Mikkelsen, K.; Stojanovska, L.; Apostolopoulos, V. The Effects of Vitamin B in Depression. Curr. Med. Chem. 2016, 23, 4317-4337. [CrossRef]

25. Milaneschi, Y.; Bandinelli, S.; Penninx, B.W.; Corsi, A.M.; Lauretani, F.; Vazzana, R.; Semba, R.D.; Guralnik, J.M.; Ferrucci, L. The relationship between plasma carotenoids and depressive symptoms in older persons. World J. Biol. Psychiatry 2012, 13, 588-598. [CrossRef] [PubMed]

26. Mrug, S.; Orihuela, C.; Mrug, M.; Sanders, P.W. Sodium and potassium excretion predict increased depression in urban adolescents. Physiol. Rep. 2019, 7, e14213. [CrossRef] [PubMed]

27. Sureda, A.; Tejada, S. Polyphenols and depression: From chemistry to medicine. Curr. Pharm. Biotechnol. 2015, 16, 259-264. [CrossRef] [PubMed]

28. Lang, U.E.; Beglinger, C.; Schweinfurth, N.; Walter, M.; Borgwardt, S. Nutritional aspects of depression. Cell Physiol. Biochem. 2015, 37, 1029-1043. [CrossRef] [PubMed]

29. Smith, A.P.; Rogers, R. Positive effects of a healthy snack (fruit) versus an unhealthy snack (chocolate/crisps) on subjective reports of mental and physical health: A preliminary intervention study. Front. Nutr. 2014, 1, 10. [CrossRef] [PubMed]

30. Moher, D.; Liberati, A.; Tetzlaff, J.; Altman, D.G. PRISMA Group. Preferred reporting items for systematic reviews and meta-analyses: The PRISMA statement. PLoS Med. 2009, 6, e1000097. [CrossRef] [PubMed]

31. Assessing Risk of Bias in Non-Randomized Studies. Chapter 13.5.2.3. Available online: http://handbook-5-1. cochrane.org/ (accessed on 31 October 2019).

32. Wells, G.A.; Shea, B.; O'Connell, D.; Peterson, J.; Welch, V.; Losos, M.; Tugwell, P. The Newcastle-Ottawa Scale (NOS) for Assessing the Quality of Nonrandomised Studies in Meta-Analyses. Available online: http://www.ohri.ca/programs/clinical_epidemiology/oxford.asp (accessed on 31 October 2019).

33. Luchini, C.; Stubbs, B.; Solmi, M.; Veronese, N. Assessing the quality of studies in meta-analyses: Advantages and limitations of the Newcastle Ottawa Scale. World J. Meta-Anal. 2017, 5, 80-84. [CrossRef]

34. Lo, C.K.; Mertz, D.; Loeb, M. Newcastle-Ottawa Scale: Comparing reviewers' to authors' assessments. BMC Med. Res. Methodol. 2014, 14, 45. [CrossRef]

35. Pengpid, S.; Peltzer, K. Association between fruit/vegetable consumption and mental-health-related quality of life, major depression, and generalized anxiety disorder: A longitudinal study in Thailand. Iran. J. Psychiatry Behav. Sci. 2019, 13, 88246. [CrossRef]

36. Tung, H.H.; Tseng, L.H.; Wei, J.; Lin, C.H.; Wang, T.J.; Liang, S.Y. Food pattern and quality of life in metabolic syndrome patients who underwent coronary artery bypass grafting in Taiwan. Eur. J. Cardiovasc. Nurs. 2011, 10, 205-212. [CrossRef]

37. Whitaker, K.M.; Sharpe, P.A.; Wilcox, S.; Hutto, B.E. Depressive symptoms are associated with dietary intake but not physical activity among overweight and obese women from disadvantaged neighborhoods. Nutr. Res. 2014, 34, 294-301. [CrossRef] [PubMed]

38. Bhattacharyya, M.; Marston, L.; Walters, K.; D'Costa, G.; King, M.; Nazareth, I. Psychological distress, gender and dietary factors in South Asians: A cross-sectional survey. Public Health Nutr. 2014, 17, 1538-1546. [CrossRef] [PubMed]

39. Chai, W.; Nigg, C.R.; Pagano, I.S.; Motl, R.W.; Horwath, C.; Dishman, R.K. Associations of quality of life with physical activity, fruit and vegetable consumption, and physical inactivity in a free living, multiethnic population in Hawaii: A longitudinal study. Int. J. Behav. Nutr. Phys. Act. 2010, 7, 83. [CrossRef] [PubMed]

40. Chang, M.W.; Tan, A.; Schaffir, J. Relationships between stress, demographics and dietary intake behaviours among low-income pregnant women with overweight or obesity. Public Health Nutr. 2019, 22, 1066-1074. [CrossRef] [PubMed]

41. Cheng, H.Y.; Shi, Y.X.; Yu, F.N.; Zhao, H.Z.; Zhang, J.H.; Song, M. Association between vegetables and fruits consumption and depressive symptoms in a middle-aged Chinese population: An observational study. Medicine 2019, 98, 15374. [CrossRef] [PubMed] 
42. Gehlich, K.H.; Beller, J.; Lange-Asschenfeldt, B.; Köcher, W.; Meinke, M.C.; Lademann, J. Consumption of fruits and vegetables: Improved physical health, mental health, physical functioning and cognitive health in older adults from 11 European countries. Aging Ment. Health 2019, 7, 1-8. [CrossRef]

43. Gehlich, K.H.; Beller, J.; Lange-Asschenfeldt, B.; Köcher, W.; Meinke, M.C.; Lademann, J. Fruit and vegetable consumption is associated with improved mental and cognitive health in older adults from non-Western developing countries. Public Health Nutr. 2019, 22, 689-696. [CrossRef]

44. Goh, C.M.J.; Abdin, E.; Jeyagurunathan, A.; Shafie, S.; Sambasivam, R.; Zhang, Y.J.; Vaingankar, J.A.; Chong, S.A.; Subramaniam, M. Exploring Singapore's consumption of local fish, vegetables and fruits, meat and problematic alcohol use as risk factors of depression and subsyndromal depression in older adults. BMC Geriatr. 2019, 19, 161. [CrossRef]

45. Ocean, N.; Howley, P.; Ensor, J. Lettuce be happy: A longitudinal UK study on the relationship between fruit and vegetable consumption and well-being. Soc. Sci. Med. 2019, 222, 335-345. [CrossRef]

46. Salvatore, F.P.; Relja, A.; Simunovic Filipcic, I.; Polasek, O.; Kolcic, I. Mediterranean diet and mental distress: 10,001 Dalmatians study. Mediterranean diet and mental distress: 10,001 Dalmatians study. Br. Food J. 2019, 121, 1314-1326. [CrossRef]

47. Azupogo, F.; Seidu, J.A.; Issaka, Y.B. Higher vegetable intake and vegetable variety is associated with a better self-reported health-related quality of life (HR-QoL) in a cross-sectional survey of rural northern Ghanaian women in fertile age. BMC Public Health 2018, 18, 920. [CrossRef] [PubMed]

48. Baharzadeh, E.; Siassi, F.; Qorbani, M.; Koohdani, F.; Pak, N.; Sotoudeh, G. Fruits and vegetables intake and its subgroups are related to depression: A cross-sectional study from a developing country. Ann. Gen. Psychiatry 2018, 17, 46. [CrossRef] [PubMed]

49. Boehm, J.K.; Soo, J.; Zevon, E.S.; Chen, Y.; Kim, E.S.; Kubzansky, L.D. Longitudinal associations between psychological well-being and the consumption of fruits and vegetables. Health Psychol. 2018, 37, 959-967. [CrossRef] [PubMed]

50. Brookie, K.L.; Best, G.I.; Conner, T.S. Intake of Raw Fruits and Vegetables Is Associated with Better Mental Health Than Intake of Processed Fruits and Vegetables. Front. Psychol. 2018, 9, 487. [CrossRef] [PubMed]

51. Hoare, E.; Hockey, M.; Ruusunen, A.; Jacka, F.N. Does Fruit and Vegetable Consumption During Adolescence Predict Adult Depression? A Longitudinal Study of US Adolescents. Front. Psychiatry 2018, 9, 581. [CrossRef]

52. Jyväkorpi, S.K.; Urtamo, A.; Pitkala, K.H.; Strandberg, T.E. Happiness of the oldest-old men is associated with fruit and vegetable intakes. Eur. Geriatr. Med. 2018, 9, 687-690. [CrossRef]

53. Pagliai, G.; Sofi, F.; Vannetti, F.; Caiani, S.; Pasquini, G.; Lova, R.M.; Cecchi, F.; Sorbi, S.; Macchi, C. Mediterranean diet, food consumption and risk of late-life depression: The Mugello Study. J. Nutr. Health Aging 2018, 2, 569-574. [CrossRef]

54. Saghafian, F.; Malmir, H.; Saneei, P.; Keshteli, A.H.; Hosseinzadeh-Attar, M.J.; Afshar, H.; Siassi, F.; Esmaillzadeh, A.; Adibi, P. Consumption of fruit and vegetables in relation with psychological disorders in Iranian adults. Eur. J. Nutr. 2018, 57, 2295-2306. [CrossRef]

55. Tan, S.L.; Storm, V.; Reinwand, D.A.; Wienert, J.; de Vries, H.; Lippke, S. Understanding the Positive Associations of Sleep, Physical Activity, Fruit and Vegetable Intake as Predictors of Quality of Life and Subjective Health Across Age Groups: A Theory Based, Cross-Sectional Web-Based Study. Front. Psychol. 2018, 9, 977. [CrossRef]

56. Welch, J.D.; Ellis, E.M. Sex Differences in the Association of Perceived Ambiguity, Cancer Fatalism, and Health-Related Self-Efficacy with Fruit and Vegetable Consumption. J. Health Commun. 2018, 23, 984-992. [CrossRef]

57. Bishwajit, G.; O’Leary, D.P.; Ghosh, S.; Sanni, Y.; Shangfeng, T.; Zhanchun, F. Association between depression and fruit and vegetable consumption among adults in South Asia. BMC Psychiatry 2017, 17, 15. [CrossRef] [PubMed]

58. Nguyen, B.; Ding, D.; Mihrshahi, S. Fruit and vegetable consumption and psychological distress: Cross-sectional and longitudinal analyses based on a large Australian sample. BMJ Open 2017, 7, 014201. [CrossRef] [PubMed]

59. Peltzer, K.; Pengpid, S. Dietary consumption and happiness and depression among university students: A cross-national survey. J. Psychol. Afr. 2017, 27, 372-377. [CrossRef] 
60. Ribeiro, S.M.L.; Malmstrom, T.K.; Morley, J.E.; Miller, D.K. Fruit and vegetable intake, physical activity, and depressive symptoms in the African American Health (AAH) study. J. Affect. Disord. 2017, 220, 31-37. [CrossRef] [PubMed]

61. Richard, A.; Rohrmann, S.; Vandeleur, C.L.; Lasserre, A.M.; Strippoli, M.F.; Eichholzer, M.; Glaus, J.; Marques-Vidal, P.; Vollenweider, P.; Preisig, M. Adherence to dietary recommendations is not associated with depression in two Swiss population-based samples. Psychiatry Res. 2017, 252, 310-318. [CrossRef]

62. Warner, R.M.; Frye, K.; Morrell, J.S.; Carey, G. Fruit and Vegetable Intake Predicts Positive Affect. J. Happiness Stud. 2017, 18, 809-826. [CrossRef]

63. Wolniczak, I.; Cáceres-DelAguila, J.A.; Maguiña, J.L.; Bernabe-Ortiz, A. Fruits and vegetables consumption and depressive symptoms: A population-based study in Peru. PLoS ONE 2017, 12, 0186379. [CrossRef]

64. Chi, S.H.; Wang, J.Y.; Tsai, A.C. Combined association of leisure-time physical activity and fruit and vegetable consumption with depressive symptoms in older Taiwanese: Results of a national cohort study. Geriatr. Gerontol. Int. 2016, 16, 244-251. [CrossRef]

65. Lesani, A.; Mohammadpoorasl, A.; Javadi, M.; Esfeh, J.M.; Fakhari, A. Eating breakfast, fruit and vegetable intake and their relation with happiness in college students. Eat. Weight Disord. 2016, 21, 645-651. [CrossRef]

66. Mujcic, R.; Oswald, J.A. Evolution of Well-Being and Happiness after Increases in Consumption of Fruit and Vegetables. Am. J. Public Health 2016, 106, 1504-1510. [CrossRef]

67. Beezhold, B.; Radnitz, C.; Rinne, A.; DiMatteo, J. Vegans report less stress and anxiety than omnivores. Nutr. Neurosci. 2015, 18, 289-296. [CrossRef] [PubMed]

68. Conner, T.S.; Brookie, K.L.; Richardson, A.C.; Polak, M.A. On carrots and curiosity: Eating fruit and vegetables is associated with greater flourishing in daily life. Br. J. Health Psychol. 2015, 20, 413-427. [CrossRef] [PubMed]

69. Kingsbury, M.; Dupuis, G.; Jacka, F.; Roy-Gagnon, M.H.; McMartin, S.E.; Colman, I. Associations between fruit and vegetable consumption and depressive symptoms: Evidence from a national Canadian longitudinal survey. J. Epidemiol. Community Health 2016, 70, 155-161. [CrossRef] [PubMed]

70. Kwon, S.C.; Wyatt, L.C.; Kranick, J.A.; Islam, N.S.; Devia, C.; Horowitz, C.; Trinh-Shevrin, C. Physical activity, fruit and vegetable intake, and health-related quality of life among older Chinese, Hispanics, and Blacks in New York City. Am. J. Public Health 2015, 105, 544-552. [CrossRef] [PubMed]

71. Papier, K.; Ahmed, F.; Lee, P.; Wiseman, J. Stress and dietary behaviour among first-year university students in Australia: Sex differences. Nutrition 2015, 31, 324-330. [CrossRef]

72. Richard, A.; Rohrmann, S.; Vandeleur, C.L.; Mohler-Kuo, M.; Eichholzer, M. Associations between fruit and vegetable consumption and psychological distress: Results from a population-based study. BMC Psychiatry 2015, 15, 213. [CrossRef]

73. El Ansari, W.; Adetunji, H.; Oskrochi, R. Food and mental health: Relationship between food and perceived stress and depressive symptoms among university students in the United Kingdom. Cent. Eur. J. Public Health. 2014, 22, 90-97. [CrossRef]

74. Mihrshahi, S.; Dobson, A.J.; Mishra, G.D. Fruit and vegetable consumption and prevalence and incidence of depressive symptoms in mid-age women: Results from the Australian longitudinal study on women's health. Eur. J. Clin. Nutr. 2015, 69, 585-591. [CrossRef]

75. Rutledge, T.; Kenkre, T.S.; Thompson, D.V.; Bittner, V.A.; Whittaker, K.; Eastwood, J.A.; Eteiba, W.; Cornell, C.E.; Krantz, D.S.; Pepine, C.J.; et al. Depression, dietary habits, and cardiovascular events among women with suspected myocardial ischemia. Am. J. Med. 2014, 127, 840-847. [CrossRef]

76. Akbaraly, T.N.; Sabia, S.; Shipley, M.J.; Batty, G.D.; Kivimaki, M. Adherence to healthy dietary guidelines and future depressive symptoms: Evidence for sex differentials in the Whitehall II study. Am. J. Clin. Nutr. 2013, 97, 419-427. [CrossRef]

77. McMartin, S.E.; Jacka, F.N.; Colman, I. The association between fruit and vegetable consumption and mental health disorders: Evidence from five waves of a national survey of Canadians. Prev. Med. 2013, 56, 225-230. [CrossRef] [PubMed]

78. Meyer, B.J.; Kolanu, N.; Griffiths, D.A.; Grounds, B.; Howe, P.R.; Kreis, I.A. Food groups and fatty acids associated with self-reported depression: An analysis from the Australian National Nutrition and Health Surveys. Nutrition 2013, 29, 1042-1047. [CrossRef] [PubMed] 
79. Niu, K.; Guo, H.; Kakizaki, M.; Cui, Y.; Ohmori-Matsuda, K.; Guan, L.; Hozawa, A.; Kuriyama, S.; Tsuboya, T.; Ohrui, T.; et al. A tomato-rich diet is related to depressive symptoms among an elderly population aged 70 years and over: A population-based, cross-sectional analysis. J. Affect. Disord. 2013, 144, 165-170. [CrossRef] [PubMed]

80. Roohafza, H.; Sarrafzadegan, N.; Sadeghi, M.; Rafieian-Kopaei, M.; Sajjadi, F.; Khosravi-Boroujeni, H. The association between stress levels and food consumption among Iranian population. Arch. Iran. Med. 2013, 16, 145-148.

81. White, B.A.; Horwath, C.C.; Conner, T.S. Many apples a day keep the blues away-daily experiences of negative and positive affect and food consumption in young adults. Br. J. Health Psychol. 2013, 18, 782-798. [CrossRef]

82. Blanchflower, D.G.; Oswald, A.J.; Stewart-Brown, S. Is psychological well-being linked to the consumption of fruit and vegetables? Soc. Indic. Res. 2013, 114, 785-801. [CrossRef]

83. Davison, K.M.; Kaplan, B.J. Food intake and blood cholesterol levels of community-based adults with mood disorders. BMC Psychiatry 2012, 12, 10. [CrossRef]

84. Payne, M.E.; Steck, S.E.; George, R.R.; Steffens, D.C. Fruit, vegetable, and antioxidant intakes are lower in older adults with depression. J. Acad. Nutr. Diet. 2012, 112, 2022-2027. [CrossRef]

85. Tsai, A.C.; Chang, T.L.; Chi, S.H. Frequent consumption of vegetables predicts lower risk of depression in older Taiwanese-results of a prospective population-based study. Public Health Nutr. 2012, 15, 1087-1092. [CrossRef]

86. Konttinen, H.; Männistö, S.; Sarlio-Lähteenkorva, S.; Silventoinen, K.; Haukkala, A. Emotional eating, depressive symptoms and self-reported food consumption. A population-based study. Appetite 2010, 54, 473-479. [CrossRef]

87. Mamplekou, E.; Bountziouka, V.; Psaltopoulou, T.; Zeimbekis, A.; Tsakoundakis, N.; Papaerakleous, N.; Gotsis, E.; Metallinos, G.; Pounis, G.; Polychronopoulos, E.; et al. Urban environment, physical inactivity and unhealthy dietary habits correlate to depression among elderly living in eastern Mediterranean islands: The MEDIS (MEDiterranean ISlands Elderly) study. J. Nutr. Health Aging 2010, 14, 449-455. [CrossRef] [PubMed]

88. Li, Y.; Zhang, J.; McKeown, R.E. Cross-sectional assessment of diet quality in individuals with a lifetime history of attempted suicide. Psychiatry Res. 2009, 165, 111-119. [CrossRef] [PubMed]

89. Mikolajczyk, R.T.; El Ansari, W.; Maxwell, A.E. Food consumption frequency and perceived stress and depressive symptoms among students in three European countries. Nutr. J. 2009, 8, 31. [CrossRef] [PubMed]

90. Elfhag, K.; Rasmussen, F. Food consumption, eating behaviour and self-esteem among single v. married and cohabiting mothers and their 12-year-old children. Public Health Nutr. 2008, 11, 934-939. [CrossRef] [PubMed]

91. Giltay, E.J.; Geleijnse, J.M.; Zitman, F.G.; Buijsse, B.; Kromhout, D. Lifestyle and dietary correlates of dispositional optimism in men: The Zutphen Elderly Study. J. Psychosom. Res. 2007, 63, 483-490. [CrossRef] [PubMed]

92. Liu, C.; Xie, B.; Chou, C.P.; Koprowski, C.; Zhou, D.; Palmer, P.; Sun, P.; Guo, Q.; Duan, L.; Sun, X.; et al. Perceived stress, depression and food consumption frequency in the college students of China Seven Cities. Physiol. Behav. 2007, 92, 748-754. [CrossRef]

93. Kelloniemi, H.; Ek, E.; Laitinen, J. Optimism, dietary habits, body mass index and smoking among young Finnish adults. Appetite 2005, 45, 169-176. [CrossRef]

94. Sarlio-Lähteenkorva, S.; Lahelma, E.; Roos, E. Mental health and food habits among employed women and men. Appetite 2004, 42, 151-156. [CrossRef]

95. Cook, R.; Benton, D. The relationship between diet and mental-health. Person. Individ. Diff. 1993, 14, $397-403$. [CrossRef]

96. FAO/WHO Food and Agriculture Organization of the United Nations/World Health Organization. Fruit and Vegetables for Health. Report of a Joint FAO/WHO Workshop, 1-3 September 2004, Kobe, Japan. Available online: http://www.fao.org/3/a-y5861e.pdf (accessed on 31 October 2019).

97. National Health Service (NHS). 5 A Day Portion Sizes. Available online: https://www.nhs.uk/live-well/eatwell/5-a-day-portion-sizes/ (accessed on 31 October 2019). 
98. American Heart Association (AHA). Fruits and Vegetables Serving Sizes. Available online: https://www. heart.org/en/healthy-living/healthy-eating/add-color/fruits-and-vegetables-serving-sizes (accessed on 31 October 2019).

99. Better Health Foundation (PBH). Experts Recommend 5-9 Servings of Fruits \& Veggies Daily. Available online: https://fruitsandveggies.org/stories/iv-for-090611-leanne-heckenlaible (accessed on 31 October 2019).

100. Office of Disease Prevention and health Promotion (ODPHP). Build a Healthy Base. Available online: https://health.gov/dietaryguidelines/dga2000/document/build.htm (accessed on 31 October 2019).

101. Liu, R.H. Health-promoting components of fruits and vegetables in the diet. Adv. Nutr. 2013, 4, $384-392$. [CrossRef]

102. Benton, D.; Young, H.A. Role of fruit juice in achieving the 5-a-day recommendation for fruit and vegetable intake. Nutr. Rev. 2019, 77, 829-843. [CrossRef] [PubMed]

(C) 2020 by the authors. Licensee MDPI, Basel, Switzerland. This article is an open access article distributed under the terms and conditions of the Creative Commons Attribution (CC BY) license (http://creativecommons.org/licenses/by/4.0/). 